\title{
Acetylcholine-induced Retraction of an Identified Axon in the Developing Leech Embryo
}

\author{
Siegward-M. Elsas, Eda M. Kwak, ${ }^{a}$ and Gunther S. Stent \\ Department of Molecular and Cell Biology, University of California, Berkeley, California 94720
}

At early stages of embryonic development of the glossiphoniid leech, Theromyzon rude, a branch, termed MAC, of the axon of the segmentally iterated Retzius neuron extends into the anterior interganglionic connective nerve. At later stages, this branch disappears again in about $30 \%$ of the Retzius neurons in the standard midbody segments and in about $75 \%$ of the Retzius neurons in the two reproductive midbody segments.

The frequency of disappearance of the MAC branch increases to about $85 \%$ in all Retzius neurons upon exposure of the embryos to culture media containing $1 \mathrm{~mm}$ acetylcholine (ACh) and $10 \mu \mathrm{m}$ physostigmine during a sensitive period of axon outgrowth. This disappearance represents a retraction of the MAC branch to its point of origin, while other axon branches of the Retzius neuron remained unaffected. In later development, the retracted (medial) MAC branch was replaced by a new (lateral) branch termed LAC. The observations were made using confocal microscopy of fixed embryos stained with anti-5-HT antibody and confirmed by Lucifer yellow injection of individual Retzius neurons.

The specific retraction of a single axon branch might be attributable to the local presence of extracellular matrix molecules in the ganglionic neuropil, which is contacted only by the MAC axon branch and could render this branch susceptible to growth-regulating signals. Since Retzius axon morphology in standard segments of ACh-treated embryos resembled that of reproductive segments in untreated embryos, it appears possible that ACh treatment may have simulated a process that contributes to the segmental differentiation of the Retzius neuron.

[Key words: axogenesis, axon retraction, ACh, growth cone, serotonergic, Retzius neuron, leech development, Lucifer yellow, immunohistochemistry]

In neural development, initial axonal outgrowth is often excessive and imprecise, so that superfluous or misdirected axons, or their branches, are eventually pruned. Afferent neuronal activity

\footnotetext{
Received Mar. 28, 1994; revised Aug. 3, 1994; accepted Aug. 12, 1994.

We thank David R. Bentley, Corey S. Goodman, William B. Kristan, and Duncan K. Stuart for their assistance both in experimental details and for useful comments on the manuscript. This work was supported by Research Grants NS 12813 and HD 17088 from the National Institutes of Health and BNS 88-20033 and IBN 91-21366 from the National Science Foundation as well as by a grant from the Rowland Foundation.

Correspondence should be addressed to Siegward-M. Elsas, M.D., Box 0114 , Mubley lab, Department of Neurology, University of California, San Francisco, CA 94143.

a Current address: School of Medicine, University of California San Diego, La Jolla, CA 92093.

Copyright (C) 1995 Society for Neuroscience $\quad 0270-6474 / 95 / 151419-18 \$ 05.00 / 0$
}

has been found to play an important role in this pruning process (Rakic, 1988; O'Leary, 1989; Constantine-Paton et al., 1990; Shatz, 1990). Neurotransmitters have been implicated as regulatory signals for axonal death or survival by in vitro studies using cell culture (for reviews, see Lauder, 1987; Mattson, 1988; Lipton and Kater, 1989; Leslie, 1992).

During embryonic development of the leech nervous system, several types of identified neurons extend axon branches that eventually disappear or retract (Kuwada and Kramer, 1983; Wallace, 1984; Gao and Macagno, 1987a,b; Baptista and Macagno, 1988; Wolszon and Macagno, 1992). These neurons include the segmentally iterated, bilaterally paired Retzius neuron, the largest of the five types of serotonergic neurons in the leech nervous system (Glover, 1987; Stuart et al., 1987). At early developmental stages, the Retzius neurons in all midbody segments of the leech embryo extend axon branches into the intersegmental connective nerves, but in the two reproductive segments, these branches disappear again during their later development (Glover and Mason, 1986; Jellies et al., 1987; Loer and Kristan, 1987). This disappearance depends on the contact of growing peripheral axons with their segment-specific target, the reproductive organs (Jellies et al., 1987; Loer et al., 1987; Loer and Kristan, 1989; French et al., 1992). An overview of this segment-specific process of axon development in Theromyzon rude, the leech species studied here, is presented in Figure 1 . As is to be shown in the following, in the majority of the reproductive segments (M5 and M6) the initial, medial anterior connective axon branch (MAC) of the Retzius neuron is retracted and later replaced by another, lateral anterior connective axon branch (LAC).

What could be the cellular mechanism that causes retraction of the anterior connective axon branch? It seemed plausible that an excitatory neurotransmitter may play a role in this process, since depolarization of isolated Retzius neurons maintained in cell culture may cause retraction of their neurites (GrumbacherReinert and Nicholls, 1992; Neely, 1993). Since both adult and embryonic Retzius neurons respond to prolonged ACh exposure with a sustained depolarization (Kristan et al., 1993), we exposed intact embryos to ACh to study its effects on Retzius axon development.

\section{Materials and Methods}

\section{Embryos}

Leech neuroanatomy. The leech CNS is composed of a supraesophageal ganglion and a ventral nerve cord consisting of a chain of 32 segmental ganglia, or neuromeres, linked via longitudinal connective nerves (Muller et al., 1981). The rostral four neuromeres fuse to form the adult subesophageal ganglion and arc designated S1-S4 in rostrocaudal sequence. The succeeding 21 unfused midbody neuromeres form individ- 


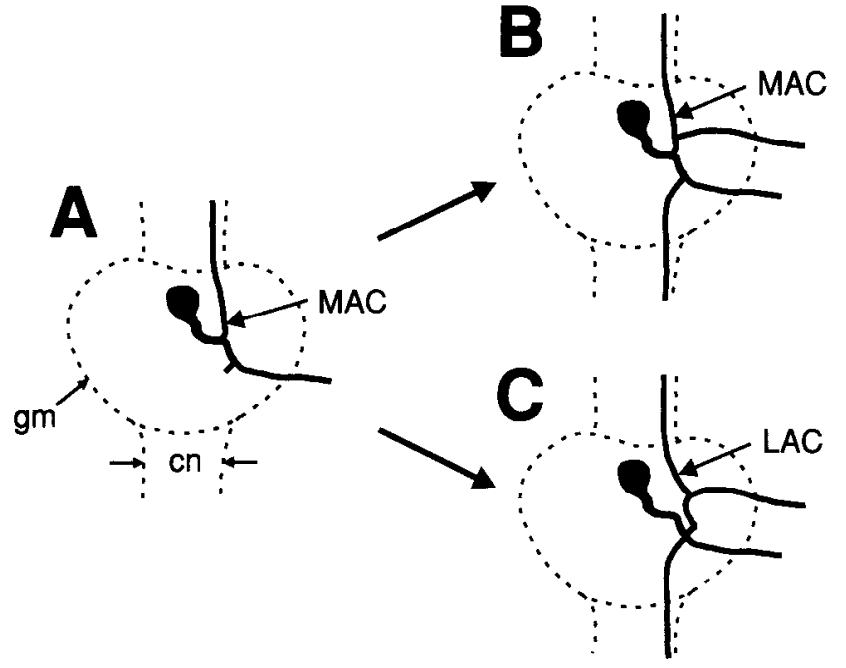

Figure 1. Schematic of Retzius axon development in a single ganglion in $T$. rude. Only the right-hand member of the Retzius neuron pair in each ganglion is shown. $M A C$, medial axon branch in the anterior connective nerve; $L A C$, lateral axon branch in the anterior connective nerve; $c n$, intersegmental connective nerve; $g m$. ganglionic margin. $A$, Early stage. The MAC axon projects into the anterior connective nerve. $B$ and $C$, Developmentally mature stage. In $B$ the MAC axon has persisted and more axon branches have appeared. This situation is predominantly found in standard segments. In $C$ the MAC axon has disappeared and has been replaced by an LAC axon. This situation is predominantly found in the reproductive segments M5 and M6. In this and all subsequent figures anterior is up.

ual ganglia and are designated M1-M21. The caudal seven neuromeres, designated $\mathrm{C} 1-\mathrm{C} 7$, fuse to constitute a caudal ganglion. We refer to segments by the designation of the neuromere they contain.

Segments M5 and M6 are termed "reproductive segments" because they contain, respectively, the male and female reproductive organs of the leech. All other, nonreproductive, midbody segments (M1-M4, M7M21) are termed "standard segments."

Culture and developmental stages. Embryos of Theromyzon rude were obtained from gravid adult specimens collected in Golden Gate Park, San Francisco. Embryos were removed from their cocoons and cultivated at $18^{\circ} \mathrm{C}$ in $\mathrm{Htr}$ culture medium $(4.8 \mathrm{mM} \mathrm{NaCl}, 1.2 \mathrm{mM} \mathrm{KCl}$, $2.0 \mathrm{mM} \mathrm{MgCl}, 8.0 \mathrm{mM} \mathrm{CaCl}_{2}$, and $1.0 \mathrm{~mm}$ sodium maleate at $\mathrm{pH} 6.6$ ), which was changed daily. Stages of embryonic development were determined according to Fernandez (1980), as modified by Kuwada and Kramer (1983). All the experiments reported here were carried out with embryos at stage 9 (which begins with the completion of coalescence of the germinal plate and lasts $4 \mathrm{~d}$ at $18^{\circ} \mathrm{C}$ ), and at stage 10 (which begins with the complete coverage of the embryo by the definitive epidermis and lasts $5 \mathrm{~d}$ at $18^{\circ} \mathrm{C}$ ). The designation of an embryo as being at stage $9(0 / 4)$ or at stage $10(2 / 5)$ indicates that the embryo had reached the first of the $4 \mathrm{~d}$ of stage 9 or the third of the $5 \mathrm{~d}$ of stage 10 , respectively.

Drug treatment. For each experiment, one batch of embryos, laid by a single mother, was randomly divided into an untreated control group and one or more treated experimental groups. At the beginning of the period of axon outgrowth [stage $9(0 / 4)$ ], the culture medium of the treated groups was replaced by $\mathrm{Htr}$ culture medium containing varying concentrations of acetylcholine and physostigmine; the culture medium of the untreated group was replaced by fresh Htr culture medium. Specimens from untreated and treated groups were fixed at various later stages.

\section{Immunohistochemistry}

The immunohistochemical procedure to visualize 5-HT neurons in the leech embryo was slightly modified from that described by Stuart et al. (1989). Embryos were preincubated for $1 \mathrm{hr}$ in culture medium containing $100 \mu \mathrm{M} 5$-HT before fixation (to increase the 5-HT content of 5-HT neurons by their 5-HT uptake systems), thereby selectively increasing their immunoreactivity and enhancing the visibility of their axons. For fixation, embryos were relaxed in ice-cold Ringer's solution $\left(130 \mathrm{~mm} \mathrm{NaCl}, 8.0 \mathrm{~mm} \mathrm{KCl}, 1.8 \mathrm{~mm} \mathrm{CaCl} 2,1.8 \mathrm{mM} \mathrm{MgSO}_{4}, 10.0 \mathrm{~mm}\right.$ Na HEPES, $\mathrm{pH} 7.4$ ) containing $8 \%$ ethanol, pinned on Sylgard, and fixed for $18-24 \mathrm{hr}$ at $4^{\circ} \mathrm{C}$ in $20 \mathrm{mg} / \mathrm{ml}$ paraformaldehyde in $50 \mathrm{~mm} \mathrm{Na}$ Hepes buffer, $\mathrm{pH}$ 7.4. After fixation, the embryos were rinsed several times and stored at $4^{\circ} \mathrm{C}$ in HBS (HEPES buffered saline; $50 \mathrm{~mm} \mathrm{Na}$ HEPES, pH 7.4, $145 \mathrm{~mm} \mathrm{NaCl}$, and $15 \mathrm{~mm}$ sodium azide). Fixed embryos were dissected with sharpened insect pins; a longitudinal incision was made and the yolk removed.

Dissected embryos were incubated for $48-72 \mathrm{hr}$ at $4^{\circ} \mathrm{C}$ in HBS containing $2 \mathrm{mg} / \mathrm{ml}$ Triton X-100, $30 \mathrm{mg} / \mathrm{ml}$ goat serum, $100 \mathrm{mg} / \mathrm{ml} \mathrm{BSA}$, and rabbit antiserum directed against a paraformaldehyde-fixed 5-HT/ BSA complex (Immunonuclear, lot 8843026, diluted 1:500). The embryos were then rinsed for several hours in the same solution without antiserum (rinse solution), and incubated for $48-72 \mathrm{hr}$ in rinse solution containing fluorescein-labeled goat anti-rabbit antibodies (high-fluorescent, Antibodies Inc., lot 9F $39 \mathrm{~W}$ ), diluted 1:400. Subsequently, the embryos were rinsed, first in rinse solution, then in HBS, and stained with the DNA-specific fluorescent dye Hoechst 33258 (Aldrich). Embryos stained by immunohistochemistry were cleared and whole mounted between a glass slide and a cover slip in a solution of $80 \%$ glycerol and $20 \% 0.1 \mathrm{M}$ Tris- $\mathrm{HCl}, \mathrm{pH} 9.0$, containing $40 \mathrm{mg} / \mathrm{ml} n$-propyl gallate. Preincubation of the antiserum solution with $40 \mu \mathrm{g} / \mathrm{ml}$ 5-HT/BSA complex before use completely eliminated the staining pattern.

\section{Lucifer yellow injections}

Lucifer yellow injections were used to visualize axon morphology at latcr stages of Retzius ncuron development, when axons of 5-IIT neurons overlap and become difficult to distinguish immunohistochemically. Lucifer yellow injections were made in Retzius neurons from standard segments M8-M12 to avoid segment-specific abnormalities.

Live stage 10 or 11 embryos were relaxed in ice-cold Ringer's solution containing $8 \%$ ethanol and pinned on Sylgard on a glass slide. A longitudinal incision of the dorsal body wall was made with fine scissors and the yolk was carefully removed. After a longitudinal incision of the visceral mesoderm, the embryos were turned over and repinned, and a longitudinal incision of the ventral body wall was made to expose the embryonic nerve cord and ganglia.

Dissected embryos were continually superfused with ice-cold Ringer's solution containing $10 \mathrm{~mm}$ glucose. Neurons were visualized by Nomarski differential interference contrast microscopy, using a Zeiss Achromat $40 / 0.75 \mathrm{~W} 40 \times$ water-immersion objective on a Zeiss microscope. A 7\% solution of the lithium salt of Lucifer yellow (Sigma) in doubly distilled water was iontophoresed into Retzius neurons, using glass electrodes of $120-200 \mathrm{M} \Omega$ resistance and several $30 \mathrm{sec}$ pulses of $0.5 \mathrm{nA}$ hyperpolarizing current.

After brief immediate inspection under epifluorescence microscopy with Zeiss filter set 487705 the embryos were fixed in $4 \%$ paraformaldehyde at $12^{\circ} \mathrm{C}$ for $1-2.5 \mathrm{hr}$. After rinsing with cold Ringer's solution, the embryos were immediately scanned under the confocal microscope using the same objective.

\section{Microscopy}

Immunohistochemically stained embryos were examined under epifluorescence microscopy; Zeiss filter sets 487701 and 487717 were used to visualize the fluorescence of Hoechst 33258 and fluorescein, respectively.

Both immunohistochemically stained and Lucifer yellow-injected embryos were scanned under an MRC-600 argon laser confocal system (Bio-Rad) attached to a Zeiss Axioplan microscope. Optical sections (18-30) separated by $1.08 \mu \mathrm{m}$ were made from each specimen. In some of the Lucifer yellow-injected specimens, some optical sections contained Lucifer yellow that had leaked out of the Retzius neuron. Such areas were removed from the individual sections containing them to make underlying axons visible in the combined image.

After enhancement of contrast, optical sections were combined to a single image. In the black and white micrographs, the images were electronically inverted to negative images. In the color micrographs, false colors were generated by assigning colors to brightness values. Images were then printed by a thermal dye sublimation printer, Kodak Excel/7700. 


\section{Results}

Normal axon development of the Retzius neuron in $\mathrm{T}$. rude Morphology. The morphology typical of developmentally mature, Lucifer yellow-injected Retzius neurons in midbody ganglia of stage $10(3 / 5) T$. rude embryos is shown in Figure 2, $A$ and $B$. The Retzius neuron is monopolar. Projecting one primary axon which branches in the ganglionic neuropil, it sends peripheral axonal branches into the ipsilateral segmental nerves and interganglionic axonal branches into the ipsilateral connective nerves. The peripheral axonal branches following the DP (dorsoposterior) and MA (medioanterior) peripheral nerves will be referred to as the DP and MA axon, respectively. In some Retzius neurons, additional peripheral axonal branches following the AA (anteroanterior) (e.g., Fig. 2C) or the PP (posteroposterior) peripheral nerves may be present as well.

One interganglionic axonal branch (designated the PC axon) is projected into the posterior connective nerve and another, which can be of two types with respect to its branching pattern, is projected into the anterior connective nerve. One type (designated the MAC axon) arises at a medial site of the primary axon (Fig. 2A), while the other type (designated the LAC axon) arises at a lateral site (Fig. $2 B, C$ ). Figure $2 C$ shows the full extent of a Retzius LAC axon in an embryo of the larger leech, Placobdella parasitica, where the axon branches are easier to visualize by Lucifer yellow injection. In most embryos, both types of branching patterns of Retzius neurons are present. The two paired Retzius neurons in the same ganglion may have different types of branching patterns, with both types appearing to be randomly distributed among segments (except for the reproductive segments), with a uniform frequency among embryos of the same batch. Table 1 presents the relative trequency of different types of branching patterns in a representative batch of embryos. This frequency varies between different embryo batches (data not shown). In the sample surveyed here, $68 \%$ of all Retzius neurons in standard segments have an MAC axon and failed to grow an LAC axon, while $20 \%$ lacked an MAC axon and grew an LAC axon. The remainder is accounted for by Retzius neurons that display both MAC and LAC (2\%) or neither MAC nor LAC axons (10\%). As will be documented further below, in the first case neither axon reaches into the anterior connective nerve, and both of these cases represent transient developmental stages of Retzius neurons that ultimately will have only an LAC axon.

The axonal morphology of Retzius neurons at stage 10(3/5) was chosen as the endpoint of developmental observation. At this stage, the Retzius neurons have established their definitive axonal branching pattern and have taken on most of their adult characteristics. Both PC axons, as well as MAC or LAC axons, have entered adjacent posterior or anterior ganglia, respectively, and project into the periphery via the segmental nerve roots of these ganglia.

Disappearance of MAC axons from some Retzius neurons. As is to be shown in the following, the difference between the two types of axonal branching patterns in Retzius neurons reflects their alternative developmental pathways. While the axonal pattern of all Retzius neurons in $T$. rude is similar for the first few days of their development, about $4.5 \mathrm{~d}$ after the soma of the serotonergic Retzius neuron first becomes discernible by immunohistochemical staining with anti-5-HT antibody, the MAC axon of some Retzius neurons disappears from the stained nerve cord. Thereupon, the original MAC axon on the medial route is replaced by an LAC axon which grows into the anterior connective nerve along a more lateral route through the ganglionic neuropil. The LAC axon is identifiable in immunohistochemically stained embryos by the characteristic U-shape of its initial sector. During the transition from one axonal pattern to the other, both MAC and LAC axons can often be seen to project from the same neuron, but at their developmental end point, Retzius neurons show either an MAC axon or an LAC axon, and very rarely both.

Immunohistochemistry of developing nerve cords. Most of the morphological data regarding the axonal development of Retzius neurons presented here were obtained from embryos fixed during stages 9 and 10 and stained immunohistochemically with anti-5-HT antibody. Pairs of stained Retzius neurons are apparent in midbody segments of stage $9(2 / 4)$ embryos. In most segments, the Retzius primary axon has already branched to form the two arms of a $\mathrm{T}$ junction (Fig. 3A). An MAC axon forms the anteriorly projecting branch and the extension of the primary axon forms the posterior branch of the T. DP axons begin to project into the periphery and an additional PC axon projecting posteriorly is sometimes present. Large swellings, or growth cones, with numerous filopodia, are frequently prominent at the growing ends of the MAC and DP axons.

The MAC axons have extended further anteriorly in stained Retzius neurons in the midbody segments of stage 9(3/4) embryos. Growth cones are still present at the MAC axon tips, but their surface area is smaller than in the stage 9(2/4) embryos (Fig. $3 B$ ). The DP axon and the PC axon (if present) are also more extended at this time. The variability in the time of onset of outgrowth of the PC axons will be documented further below (see Fig. 9B).

In midbody ganglia of stained stage $10(0 / 5)$ embryos, in addition to the Retzius neurons, the somata and axons of three other paired serotonergic neurons, designated vls, dls, and pms, have become immunoreactive to the 5-HT antibody and are discernible by their staining in the posterior and lateral parts of the two adjacent segmental ganglia (Fig. 3D). Their axonal tracts course more dorsally, however, and are clearly distinguishable from the Retzius axons (Stuart et al., 1989). The anterior-growing MAC axons and the posterior-growing PC axons have fasciculated in the connective nerves linking the ganglia and no LAC axons are present (Fig. $3 D$ ).

In midbody ganglia of stained stage $10(1 / 5)$ embryos, the Retzius neurons now stain less intensely than the serotonergic vls and dls neurons, and MAC axons are still present and LAC axons are absent (Fig. $3 F$ ).

Retzius neurons in reproductive segments. The neuronal morphology of the ganglia in the reproductive segments M5 and M6, which contain the leech reproductive organs, differs in several respects from that in the ganglia of the other, standard midbody segments, including soma size and axonal projection pattern and connectivity of mature Retzius neurons (Glover and Mason, 1986; Jellies et al, 1987; Loer et al., 1987; Baptista et al., 1990; Wittenberg et al., 1990). In immunohistochemically stained stage $10(1 / 5)$ embryos, the 5-HT immunostaining of Retzius somata in the reproductive segments is more intense and the local branching of the peripheral axons more extensive than in the standard segments in the same specimen (Fig. 4A) or in the standard segments of comparable specimens (Fig. $3 F$ ). At this stage, Retzius neurons in the reproductive segments can show both MAC and LAC axons (right two Retzius neurons in Fig. 4A). In stained stage 10(2/5) embryos, most Retzius neurons 

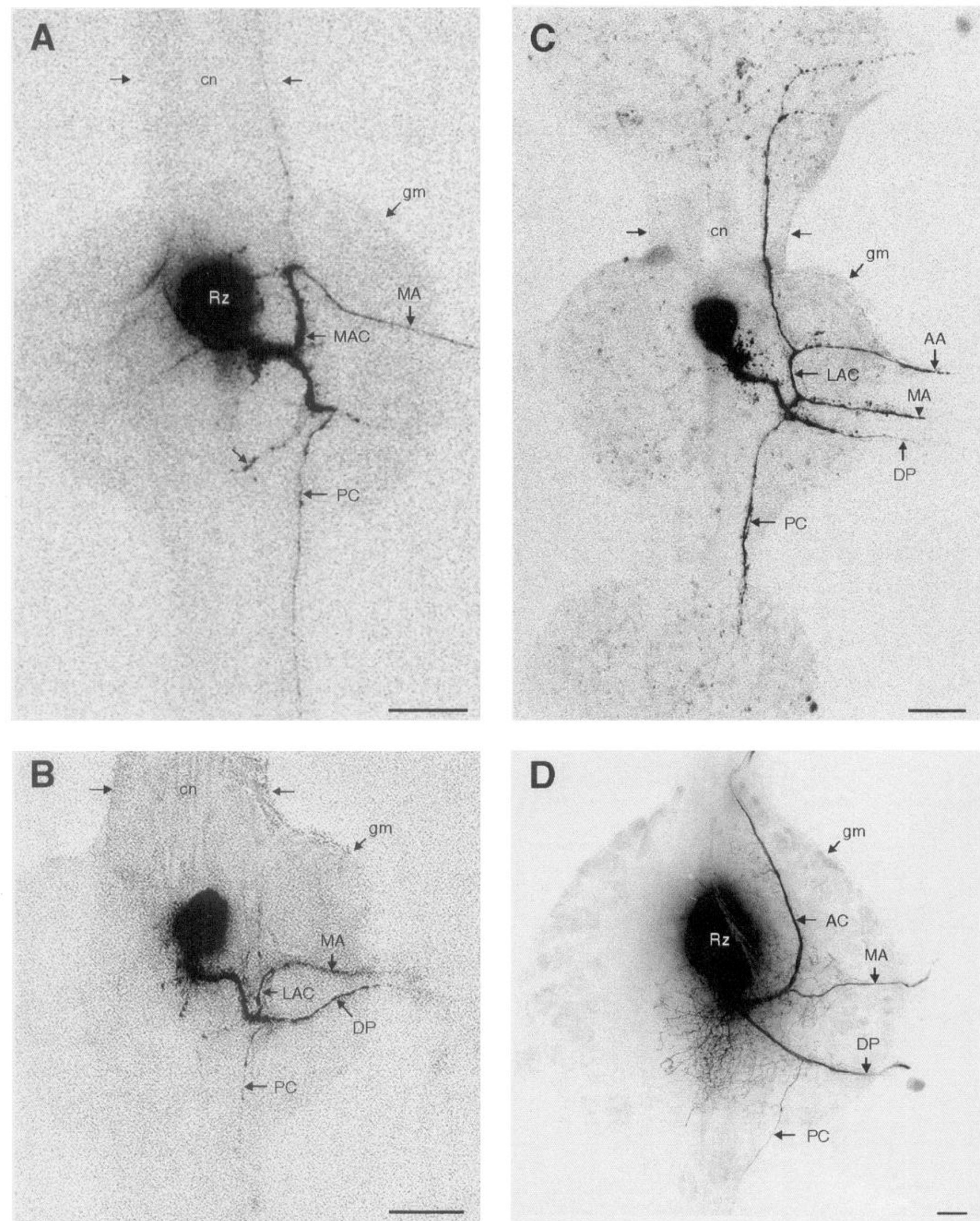

Figure 2. Lucifer yellow-injected Retzius neurons in midbody ganglia. $A-C$ present confocal micrographs of embryonic Retzius neurons and $D$ is an epifluorescent micrograph of a mature Retzius neuron. $A$, Retzius neuron (dark) with an MAC axon in a stage $11 T$. rude, normalized age $t_{\mathrm{R}}$ about $12 \mathrm{~d}$. The unlabeled arrow points to a transient, medial posterior axon branch of the Retzius neuron, which is not seen in mature neurons. It is also visible in $B$ and in Figure $3 A . B$, Retzius neuron with an LAC axon in a stage $11 T$. rude; $t_{\mathrm{R}}$ about $12 \mathrm{~d}$. Only the initial sector of the 
in the reproductive segments have LAC axons and have lost their MAC axons (Fig. 4B).

It should be noted, however, that in contrast to LAC axons of standard segments, the LAC axons of reproductive segments often do not enter the connective nerve. Also, the 5-HT immunostaining of both somata and axons of the Retzius neurons is more intense in the reproductive than in the standard segments (Fig. 4A,B). This increase in the intensity of 5-HT immunostaining at the time of loss of MAC axons and gain of LAC axons was observed in 11 of 20 Retzius neurons in reproductive segments M5 and M6 of five embryos.

We compared MAC axon retention and LAC axon acquisition in standard and reproductive midbody segments in the same five embryos (Table 1). Whereas about $70 \%$ of the Retzius neurons retained an MAC axon and $20 \%$ acquired an LAC axon in standard midbody segments by stage $10(3 / 5)$, only $20 \%$ retained an MAC axon and about $70 \%$ acquired an LAC axon in reproductive segments. The difference between standard and reproductive segments was statistically significant $(p<0.001)$.

Normalized Retzius neuron age. It is possible to follow the progress of neuronal development in the leech by comparing homologous neurons in progressively anterior segments in the same embryo, since any segment is developmentally more advanced than the immediate posterior segment. This smooth anterior-posterior developmental gradient is attributable to the derivation of the germinal plate from blast cells sequentially generated through stem cell divisions of large teloblast blastomeres (Weisblat et al., 1980, 1984; Stent et al., 1982; Weisblat and Shankland, 1985). In the following section on Retzius neuron development, the developmental gradient is used to assign different developmental stages to different segments of a single embryo.

To quantify the developmental gradient, the set of embryos fixed for the developmental study was scored for the appearance of several characteristic features in Retzius neuron development, such as the first appearance of 5-HT immunoreactivity in the soma and first outgrowth of DP, MA, and LAC axons. The relative order of appearance of these features in any given segment was found to be the same in all midbody segments. In each specimen the most posterior (i.e., the youngest) segment was scored in which a particular feature was manifest. The scored segment numbers were then averaged for each feature and plotted against developmental time (Fig. 5A).

Linear regression lines were calculated for each data set. The similar slopes of the regression lines indicate that, except for a fixed intersegmental delay, the time course of development is the same in each segment. The magnitude of that delay, given by the reciprocal of the slope of the regression lines, amounts to $0.16 \mathrm{~d} / \mathrm{segment}$ for the midbody segments M1-M21 examined here. Head segments (S1-S4) and tail segments (C1-C7), which showed smaller and nonlinear age differences, were excluded from this analysis.

We define the normalized developmental age of a Retzius neu- ron in a midbody segment as the time elapsed since it first became discernible by anti-5-HT immunohistochemistry in an embryo preincubated with 5-HT. In analogy to a procedure put forward by Braun and Stent (1989), the normalized age $t_{\mathrm{R}}$ of a Retzius neuron in the $n$th midbody segment, is defined as

$$
t_{\mathrm{R}}=t_{\mathrm{C}}-k_{\mathrm{R}}-g_{\mathrm{T}}(n-1),
$$

where $t_{\mathrm{C}}$ is the clock time in days elapsed since the completion of coalescence of the germinal plate (onset of embryonic stage 9 ), $k_{\mathrm{R}}$ is the average time delay from the completion of coalescence until the Retzius neuron in segment Ml first becomes discernible by its 5 -HT immunoreactivity, and $g_{\mathrm{T}}$ is the developmental delay per segment at a temperature $T$. (According to the data of Fig. $5 A, g_{18^{\circ}}=0.16 \mathrm{~d} /$ segment, and $k_{\mathrm{R}}=0.67 \mathrm{~d}$, as given by the $\mathrm{x}$-intercept of the regression line $\mathrm{S}$.)

Summary of axon development. In Figure 6, $A$ and $B$ present a summary of the normal outgrowth patterns of Retzius axons at progressively later developmental ages $t_{\mathrm{R}}$. They are based upon stained Retzius neurons in embryos fixed at progressively later clock times $t_{\mathrm{C}}$, such as those presented in Figure $3 A-F$.

At $t_{\mathrm{R}}=0$, when the Retzius neuron soma becomes first discernible by 5 -HT immunoreactivity, the primary axon is already present and has bifurcated into an anterior and a posterior branch to form a $\mathrm{T}$ junction.

By $t_{\mathrm{R}}=2 \mathrm{~d}$, the posterior arm of the $\mathrm{T}$ has grown out into the peripheral DP nerve, giving rise to the DP axon. The anterior arm of the $T$ has grown along a medial route through the ganglionic neuropil and entered the anterior connective nerve, thus forming the $\mathrm{M} \Lambda \mathrm{C}$ axon.

By $t_{\mathrm{R}}=3.5 \mathrm{~d}$, the MAC axon has reached the DP axon in the adjacent anterior ganglion (Fig. 6A), and the posterior arm of the $\mathrm{T}$ has given rise to the $\mathrm{PC}$ axon, which has entered the posterior connective nerve and grown toward the adjacent posterior ganglion. However, since frontward-growing MAC and rearward-growing PC axons of adjacent ganglia meet and fasciculate (Fig. 3D), in most cases the rearward growth of PC axons in the connective nerve cannot be observed (except when the posterior MAC axon happens to be absent), as the two axons often can no longer be distinguished under immunohistochemical staining. In some Retzius neurons, the MAC axon has disappeared, and the posterior arm of the $\mathrm{T}$ has instead given rise to an LAC axon, which has grown along a more lateral route through the ganglionic neuropil (Fig. 6B).

By $t_{\mathrm{R}}=5 \mathrm{~d}$, the PC axon has entered the adjacent posterior ganglion, where it takes a more lateral route in the ganglionic neuropil than the already present MAC axon of the Retzius neuron of that ganglion. It has reached the posterior arm of the axonal $\mathrm{T}$ of that Retzius neuron at, or close to, the branch point of the DP axon and has projected into the DP peripheral nerve of the posterior segment. Likewise, the anterior connective axon (either MAC or LAC) has projected into the DP peripheral nerve of the next anterior segment.

Dynamics of axon outgrowth. The detailed dynamics of out-

LAC axon inside the ganglion, which forms the link from the DP axon to the MA axon, is visible. $C$, Retzius neuron with an LAC axon in a Placobdella parasitica embryo, $t_{\mathrm{R}}$ equivalent to about $4.5 \mathrm{~d}$. Note the axonal projections into adjacent ganglia. $D$, Epifluorescent micrograph of a Lucifer yellow injected Retzius neuron (dark) in a mature $H$. medicinalis. The anterior connective axon (labeled $A C$ ) is similar to the MAC axon. $R z$, Retzius neuron soma; $A A$, axon in anteroanterior peripheral nerve; $D P$, axon projecting into the DP peripheral nerve; $M A$, axon in MA peripheral nerve; $M A C$, medial anterior connective axon; $L A C$, lateral anterior connective axon; $P C$, posterior connective axon; gm, ganglionic margin; $c n$, interganglionic connective nerve (between unlabeled arrows). Scale bars $20 \mu \mathrm{m}$. Images in this and the following two figures have been electronically inverted, rendering the fluorescent stain dark. 
Table 1. Percentage of Retzius neurons with MAC or LAC axons in untreated and ACh-treated embryos

\begin{tabular}{llcc} 
& & & Reproductive \\
& Axons & Standard Retzius & Retzius \\
\hline Untreated & MAC (+), LAC (-) & $68 \% \pm 5.7(72)$ & $22 \% \pm 10(4)$ \\
& MAC (-), LAC (+) & $20 \% \pm 3.3(23)$ & $73 \% \pm 8(13)$ \\
& MAC (+), LAC (+) & $2 \% \pm 1.8(3)$ & $5 \% \pm 5(1)$ \\
ACh-treated & MAC (-), LAC (-) & $10 \% \pm 3.4(12)$ & 0 \\
& MAC (+), LAC (-) & $12 \% \pm 3.4(21)$ & $5 \% \pm 5(1)$ \\
& MAC (-), LAC (+) & $83 \% \pm 3.1(143)$ & $90 \% \pm 6(18)$ \\
& MAC (+), LAC (+) & $3 \% \pm 1.7(5)$ & $5 \% \pm 5(1)$ \\
& MAC (-), LAC (-) & $2 \% \pm 1.9(3)$ & 0
\end{tabular}

Standard Retzius are neurons from ganglia M1-M4 and M7-M21; reproductive Retzius are neurons from ganglia M5 and M6; $(+)$, present; $(-)$, absent. Data are expressed as percentage of scored Retzius neurons, mean \pm SEM in five immunohistochemically stained embryos at stages ranging from $10(1 / 5)$ to $10(3 / 5)$. (Within this range of stages, results of scoring were found to be independent of normalized developmental age.) The absolute number of scored Retzius neurons is given in parentheses; the total number of neurons present was 190 in standard segments and 20 in reproductive segments. Neurons with 5-HT immunoreactivity too low to allow reliable scoring of axons were excluded. Since the intensity of 5-HT immunoreactivity is lower in untreated than in ACh-treated embryos, a larger fraction of neurons of untreated embryos were excluded. $\chi^{2}$ tests were performed according to Hassard (1991). Statistically significant differences were observed between standard and reproductive Retzius in untreated embryos $\left(p<0.001, \chi^{2}=21.71\right)$ and between untreated and ACh-treated standard Retzius $\left(p<0.001, \chi^{2}=112.42\right)$. In contrast, there was no significant difference between standard and reproductive Retzius in ACh-treated embryos ( $p$ $>0.5, x^{2}=1.52$ )

growth of Retzius axons was followed in sets of embryos similar to those presented in Figure 3. The summary drawings in Figure $6, A$ and $B$, are based on these specinens. In this experiment, the lengths of four different types of axons-DP, MAC, PC, and LAC - were individually assessed in Retzius neurons of various developmental ages $t_{\mathrm{R}}$. Since the absolute length of axons depends on the amount of stretch applied to the embryo during fixation, normalized lengths of developing axons were scored relative to the ganglionic borders. A set of discretionary categories was used to designate increasing axon lengths, defined separately for each axon type as shown in the drawings of Figures $7 A, 8 A, 9 A$, and $10 A$.

The dynamics of outgrowth of the DP axon is shown in Figure $7 B$. At $t_{\mathrm{R}}=0$, no DP axons are present, but by $t_{\mathrm{R}}=1 \mathrm{~d}$, a rapid outgrowth of DP axons is underway, and by $t_{\mathrm{R}}=1.5 \mathrm{~d}$, more than $90 \%$ of them have reached their end-point length.

By contrast, the outgrowth of the MAC axon in the population of Retzius neurons is quite asynchronous. As shown in Figure $8 B$, during $t_{\mathrm{R}}-1.5-2.5 \mathrm{~d}$, the population of MAC axons is fairly evenly distributed over all five normalized length values. Not until $t_{\mathrm{R}}=4 \mathrm{~d}$ do more than $80 \%$ of MAC axons reach their endpoint lengths.

Figure $9 B$ shows that outgrowth of most $\mathrm{PC}$ axons begins only at $t_{\mathrm{R}}=2.5 \mathrm{~d}$, with all $\mathrm{PC}$ axons reaching their end-point lengths by $t_{\mathrm{R}}=3.5 \mathrm{~d}$. However, in some cases (e.g., Fig. 3A), PC axons have already begun to grow by a $t_{\mathrm{R}}$ of less than $0.5 \mathrm{~d}$ and reached their full length at a $t_{\mathrm{R}}$ of less than $1 \mathrm{~d}$.

The data of Figure $10 B$ indicate, in accord with the data of Table 1, that only a minority of Retzius neurons develop an LAC axon, and that the LAC axons appear as the latest of the studied axons at $t_{\mathrm{R}}=2.5 \mathrm{~d}$, reaching their end point by $t_{\mathrm{R}}=4.5 \mathrm{~d}$.

In any more detailed interpretation of the Figures 8 and 10 , one must take into account, that as shown in Table 1 and Figure $4, A$ and $B$, the proportion of Retzius neurons that lose their MAC axon and acquire an LAC axon is much higher in the reproductive segments than in standard segments. For technical reasons, the two reproductive segments are underrepresented in the data pertaining to $t_{\mathrm{R}}=4 \mathrm{~d}$ and higher, and overrepresented at $t_{\mathrm{R}}=3.5 \mathrm{~d}$. Thus, the transient decrease of MAC4 axons at $t_{\mathrm{R}}$ $=3.5 \mathrm{~d}$ manifest in Figure $8 B$ is attributable to the overrepresentation of reproductive Retzius neurons, as is the transient increase of LAC2 axons at $t_{\mathrm{R}}=3.5 \mathrm{~d}$ manifest in Figure $10 \mathrm{~B}$. The apparent substantial decrease in the frequency of LAC axons at $t_{\mathrm{R}}=4 \mathrm{~d}$ and older is attributable to the underrepresentation of reproductive Retzius neurons.

\section{Retzius axon development in embryos treated with ACh and physostigmine}

The seemingly random disappearance of MAC axons in about $20 \%$ of Retzius neurons of standard midbody segments suggests that these axons are sensitive to some extrinsic factor whose activity remains just below the threshold level in most standard midbody segments but happens to reach suprathreshold levels in about one-fifth of them. In most reproductive segments, however, in whose special physiological and anatomical context Retzius neurons are known to deviate from the normal course of morphological development (Glover and Mason, 1986; Jellies et al., 1987; Loer et al., 1987; Wittenberg et al., 1990), the putative factor would be at suprathreshold level and, thus, cause the disappearance of MAC axons.

Retraction of neurites of Retzius neurons in cell culture has been observed upon electrical stimulation (Grumbacher-Reinert and Nicholls, 1992) or elevated $\mathrm{K}^{+}$(Neely, 1993). Therefore, it seemed possible that the putative factor induces the disappearance of the MAC axon by causing its depolarization, or may involve an excitatory neurotransmitter.

To test this hypothesis, we exposed $T$. rude embryos to acetylcholine (ACh) at and just before the developmental stages during which the MAC axons normally disappear, to see whether this treatment increases the proportion of Retzius neurons with disappearing MAC axons. The choice of ACh was suggested by the finding that both adult and embryonic Retzius neurons respond to prolonged cxposure to $\mathrm{ACh}$ with a sustaincd depolarization (Kristan et al., 1993).

Effects of ACh treatment on axon development. Experiments were, therefore, carried out in which stage $9(0 / 4)$ embryos were 
exposed during the period of axon outgrowth to $1 \mathrm{mM} \mathrm{ACh}$ and $10 \mu \mathrm{M}$ physostigmine (eserine), a reversible cholinesterase inhibitor, whose presence enhances acetylcholine activity. In the following account, "ACh treatment" refers to this procedure. The ACh-treated embryos were fixed at times ranging from stage $10(1 / 5)$ to $10(3 / 5)$ and scored for the presence of MAC. and LAC axons. Table 1 presents the relative frequencies of MAC and LAC axons in a representative group of ACh-treated embryos and a corresponding group of untreated embryos from the same batch. Whereas in untreated embryos $68 \%$ of Retzius neurons in standard midbody segments retain their MAC axon and $20 \%$ acquire an LAC axon, the corresponding frequencies are reversed in $\Lambda$ Ch-treated embryos, in that only $11 \%$ retain their MAC axons and $83 \%$ acquire an LAC axon. The difference between untreated and ACh-treated embryos was statistically significant $(p<0.001)$. Though less dramatic, the difference is in the same direction for Retzius neurons in reproductive segments, where even in untreated embryos only $22 \%$ of Retzius neurons retain their MAC axon and $73 \%$ acquire an LAC axon; in ACh-treated embryos, the corresponding frequencies are $5 \%$ and $90 \%$. Thus, ACh treatment greatly increases the probability that a Retzius neuron loses its MAC axon and replaces it with an LAC axon.

To examine the morphological development of Retzius axons under ACh treatment, a part of the batch of embryos studied in the experiment reported in Figures $3, A, B, D$, and $F$, was transferred at stage $9(0 / 4)$ to a medium containing $1 \mathrm{~mm} \mathrm{ACh}$ and $10 \mu \mathrm{M}$ physostigmine. The ACh-treated group of embryos was otherwise treated in the same manner as the untreated group from the same batch. The morphological development of the ACh-treated embryos is presented in Figures $3, C, E$, and $G$. In all ACh-treated embryos, the Retzius somata showed a more intense 5-HT staining than the Retzius somata of untreated embryos. The somata of other 5-HT neurons did not exhibit such a change.
At a normalized age $t_{\mathrm{R}}$ of about $1.5 \mathrm{~d}$ Retzius neurons in standard midbody segments of ACh-treated stage 9(3/4) embryos showed MAC axons lacking growth cone-like swellings with filopodia at their tips (Fig. $3 C$ ) in contrast to untreated matched embryos (Fig. $3 B$ ). Other axonal branch endings in these AChtreated embryos, for example, the rearward-growing $\mathrm{PC}$ axon (Fig. $3 C$ ), still retained growth cone-like swellings with filopodia.

At a normalized age $t_{\mathrm{R}}$ of about $2.5 \mathrm{~d}$ in ACh-treated stage $10(0 / 5)$ embryos, the MAC axons were either very short or absent altogether (Fig. 3E). LAC axons, which show the characteristic U-shape in their initial sector, had begun to appear, being longer in the developmentally more advanced anterior segments. Such LAC axons were not usually observed in the ganglia of untreated embryos (Fig. 3D).

By the normalized age $t_{\mathrm{R}}$ of about $3.5 \mathrm{~d}$ in ACh-treated stage 10(1/5) embryos, all MAC axons had disappeared and LAC axons were present in all segments including the reproductive segments M5 and M6. These LAC axons had fasciculated with the $\mathrm{PC}$ axons projecting from the next anterior ganglion (Fig. $3 G$ ).

Determination of the developmental gradient in this series of ACh-treated embryos showed that the values of the constants $k_{\mathrm{R}}$ and $g_{\mathrm{T}}$ are not significantly different from those obtained for untreated embryos of the same batch (Fig. $5 B$ ). Hence, the rate of development of Retzius neurons is not noticeably affected by ACh treatment, and the normalized Retzius neuron ages $t_{\mathrm{R}}$ are directly comparable in untreated and ACh-treated embryos.

The drawings of Figure $6 \mathrm{C}$ present a summary of the axonal outgrowth pattern under $\mathrm{ACh}$ treatment, as examined by the same procedures on which the data of Figure $6, A$ and $B$ are based. Under $\mathrm{ACh}$ treatment, the initial axonal outgrowth of DP and MAC axons follows the same course as in untreated embryos up to a normalized age $\left(t_{\mathrm{R}}\right)$ of about $1 \mathrm{~d}$. However, at an age of about $2 \mathrm{~d}$, all MAC axons in ACh-treated embryos are shorter than in untreated embryos.

\begin{abstract}
Figure 3. Confocal micrographs of midbody ganglia from untreated $(A, B, D$, and $F)$ and ACh-treated $(C, E$, and $G) T$. rude embryos, immunohistochemically stained with an anti-5-HT antibody. Images $B$ and $C, D$, and $E, F$, and $G$ each are matched pairs from untreated (left-hand image) and ACh-treated (right-hand image) embryos of a similar normalized age, which have been stained, collected, and processed under identical conditions. Cholinergic stimulation of body wall muscles presumably caused longitudinal contraction of the treated embryos, revealing more ganglia in the same field of view than in their untreated counterparts. Retzius neurons in the ACh-treated embryos in $C, E$, and $G$ show increased staining intensity. $R z$, Retzius neuron somata; $d l s, p m s, v l s$, non-Retzius serotonergic neuron somata; gm, ganglionic margin. $A$, Pair of Retzius neurons in ganglion M3 before onset of ACh treatment, at a normalized age $\left(t_{\mathrm{R}}\right)$ of about $0.5 \mathrm{~d}$; embryo stage $9(2 / 4)$. There is a large terminal swelling with numerous filopodia (GS3) at the tip of the left MAC axon (unlabeled open arrow). B. Pair of Retzius neurons in ganglion M6 of an untreated stage 9(3/4) embryo; $t_{\mathrm{R}}$ about $1.5 \mathrm{~d}$. Terminal swellings at the tips of elongating MAC axons are still present (unlabeled open arrows), but are smaller in surface area (GS2). (Retzius soma size at this early age is not influenced by segmental location.) $C$, Two pairs of Retzius neurons in ganglia M3-M4 after $24 \mathrm{hr}$ of ACh treatment; $t_{\mathrm{R}}$ about $1.5 \mathrm{~d}$, embryo stage 9(3/4). MAC axons retracted and terminal swellings absent (GS0, unlabeled filled arrow); a rearward-growing PC axon shows a normal swelling at the tip (unlabeled open arrow). The staining intensity of the Retzius neurons are increased compared to the specimen shown in $B . D$, Ganglia M4-M5 in an untreated stage $10(0 / 5)$ embryo; $t_{\mathrm{R}}$ about $2.5 \mathrm{~d}$. MAC and PC axons have fasciculated; no LAC axons present. Bilaterally paired Retzius neuron somata are superimposed on each other in this image; unlabeled open arrows mark the axonal tracts of the lateral serotonergic neurons dls and vls. $E$, Ganglia M4-M7 in an ACh-treated embryo, stage $10(0 / 5)$; $t_{\mathrm{R}}$ about $2.5 \mathrm{~d}$. MAC axons short or absent; LAC axons begin to grow, being more advanced in the developmentally older anterior (upper) segments. LAC axons show the characteristic U-shape (arrow) of its initial sector conjoined with the distal end of the Retzius primary axon. $F$, Ganglia M7-M9 in an untreated stage $10(1 / 5)$ embryo; $t_{\mathrm{R}}$ about $3.5 \mathrm{~d}$. MAC axons are present and LAC axons absent. Retzius neurons stain less intensely than dls or vls neurons. Unlabeled arrowheads mark the axonal tracts of the lateral serotonergic neurons dls and vls. G, Ganglia M6-M12 in an AChtreated stage $10(1 / 5)$ embryo; $t_{\mathrm{R}}$ about $3.5 \mathrm{~d}$. MAC axons have disappeared, replaced in all ganglia by LAC axons, which have fasciculated with $\mathrm{PC}$ axons. Retzius neurons stain more intensely than other serotonergic neurons. For $F, G$, and Figure $4, A$ and $B$, a wedge indicates the correlation of grey level to relative staining intensity to allow for a comparison of staining intensity between different neurons. Scale bars, $20 \mu \mathrm{m}$.
\end{abstract}

Figure 4. Confocal micrographs of the reproductive and adjacent ganglia in immunohistochemically stained $T$. rude embryos. A, Ganglia M5$\mathrm{M} 7$ in an untreated stage $10(1 / 5)$ embryo; the normalized age $t_{\mathrm{R}}$ is about $3.5 \mathrm{~d}$. Retzius neurons in the reproductive ganglia M5 and M6 show both MAC and LAC. axons at this stage. $B$, Ganglia M4-M7 in an untreated embryo at stage $10(2 / 5), t_{\mathrm{R}}$ about $4.5 \mathrm{~d}$. LAC axons are present in M5 and M6, but not in other ganglia; MAC axons in M5 and M6 have disappeared except in the left Retzius neuron in M5, which also lacks the increase in staining intensity seen in the other three reproductive Retzius neurons. In both specimens shown, 5-HT staining intensity of Retzius neurons in ganglia M5 and M6 is increased compared to those in ganglia M4 and M7, and their peripheral axons show extensive local branching. Scale bars, $20 \mu \mathrm{m}$. 

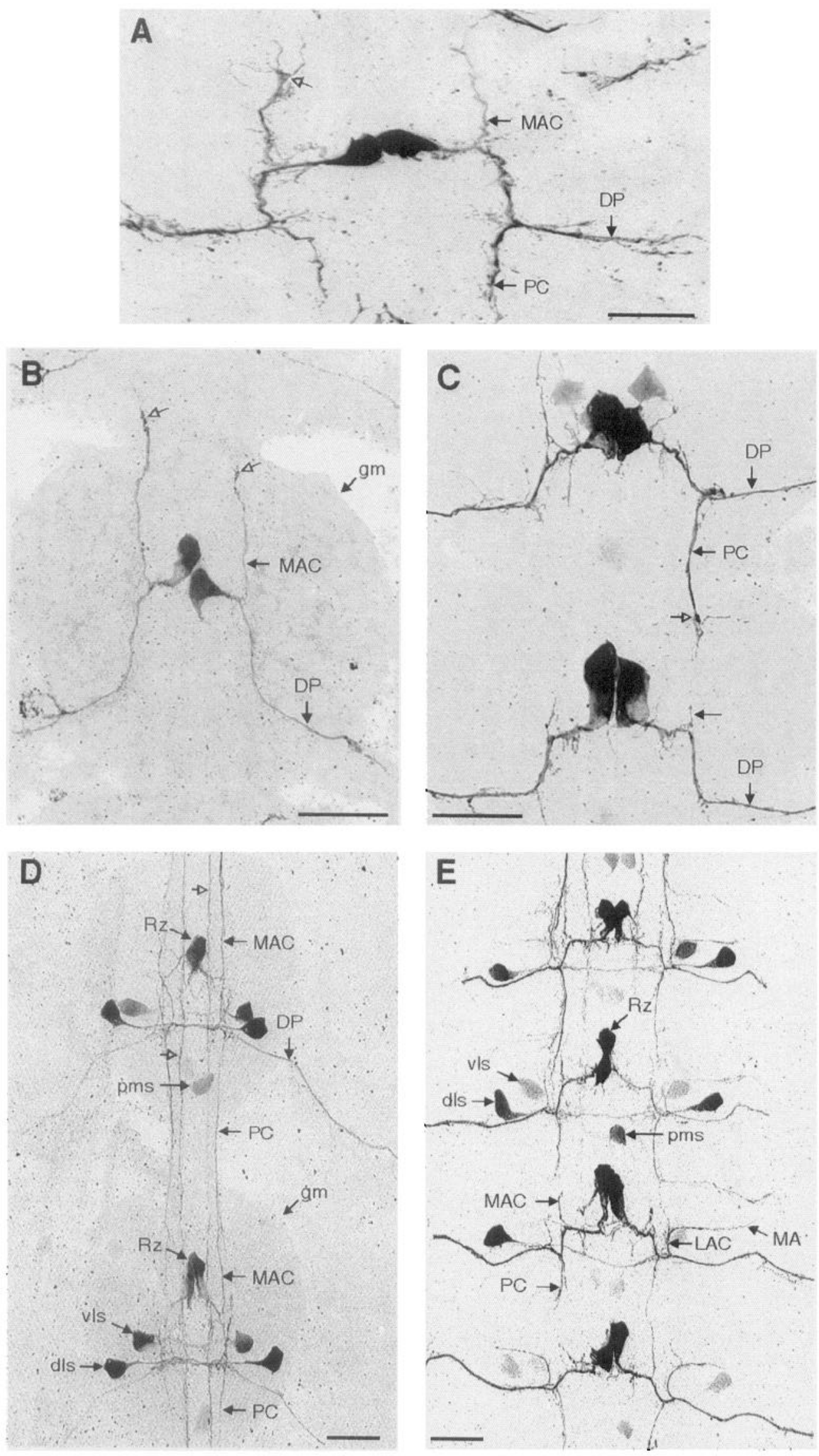

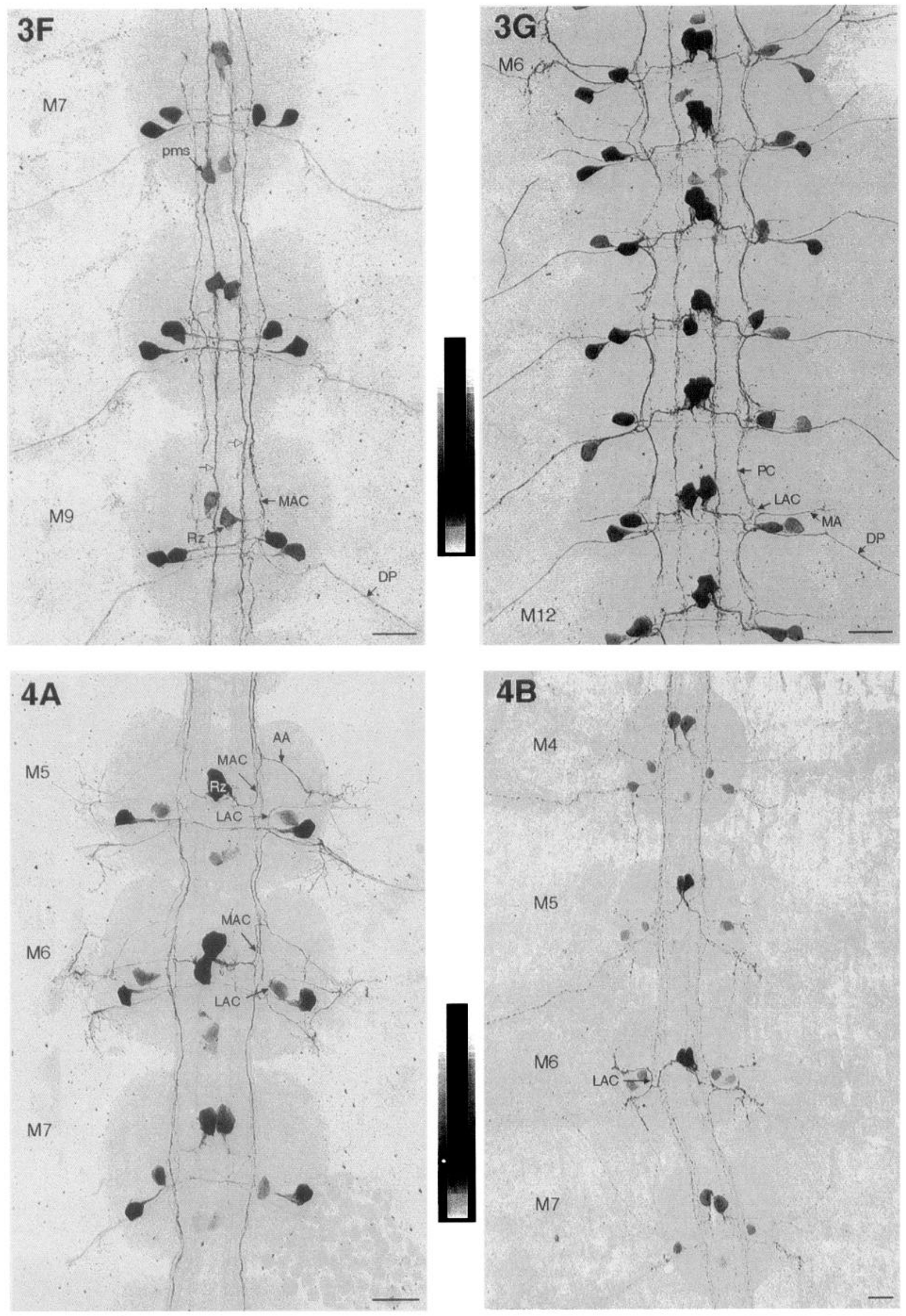
A Untreated

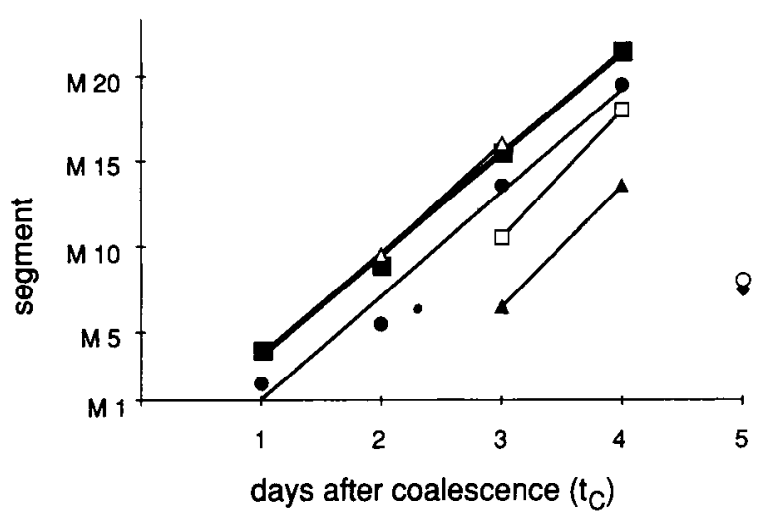

B ACh-treated

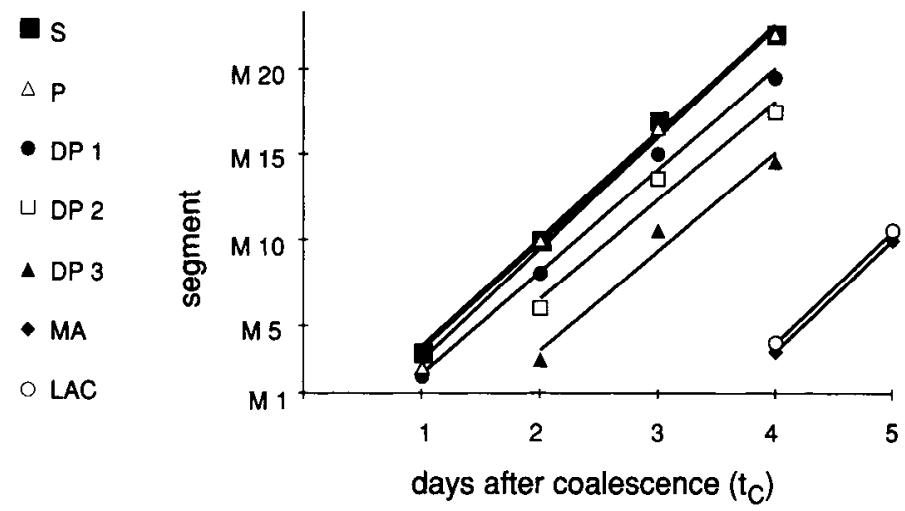

Figure 5. Quantification of the developmental gradient in Retzius neurons in untreated $(A)$ and in ACh-treated embryos $(B)$. On each of 5 consecutive days after completion of germinal plate coalescence (onset of embryonic stage 9) two to seven specimens of each group of developing embryos were fixed and scored for the presence of specific features in Retzius neuron development listed below. In each specimen, the most posterior (i.e., the youngest) midbody segment (M1-M21) in which a particular feature was manifest was scored; the scored segment numbers were then averaged for each feature. This average value was plotted against time ( $t_{c}$, days after germinal plate coalescence), and regression lines were calculated for each feature to determine linearity and the gradient of the segmental age difference. $S$, first appearance of 5-HT immunoreactivity in the soma; $P$, first outgrowth of primary posterior axon; $D P I$, first outgrowth of DP axon; $D P 2$, DP axon reaches border of ganglion; $D P 3$, DP axon reaches double length of DP2; $M A$, first outgrowth of MA axon; $L A C$, first outgrowth of LAC axon. An ANCOVA test was performed according to Hassard (1991) to compare the regression line slopes of untreated and ACh-treated embryos. The segment of first feature appearance was highly correlated to the developmental age $t_{\mathrm{C}}(p<0.001, F=1115)$; this correlation was not dependent on ACh treatment $(p>0.5, F=$ $0.927)$.

Thus, under ACh treatment, the disappearance of MAC axons begins much earlier than in untreated embryos, and well before the time MAC axons meet and fasciculate with the PC axons growing toward them. In untreated embryos, the disappearance of MAC axons occurs only after its apparent contact with the $\mathrm{PC}$ axon.

Following the disappearance of MAC axons, outgrowth of $\mathrm{I}$.AC axons is well under way by $t_{\mathrm{R}}=3.5 \mathrm{~d}$ in treated embryos. ACh treatment does not affect the later development of the DP, the PC, and MA axons, whose morphology by $t_{\mathrm{R}}=5 \mathrm{~d}$ is the same in ACh-treated as in untreated embryos.

The detailed dynamics of axonal outgrowth in embryos whose treatment was begun at stage $9(0 / 4)$ were examined by the same procedure as that applied to untreated embryos in Figures $7 B$, $8 B, 9 B$, and $10 B$. The results of this study are presented in Figures $7 C, 8 C, 9 C$, and $10 C$. The outgrowth dynamics in $\mathrm{ACh}$ treated embryos of the DP axon (Fig. $7 C$ ) and the PC axon (Fig. $9 C$ ) appeared very similar to those in untreated embryos. In contrast, a clear difference was found for the MAC axon: even though there was a similar early outgrowth to the ganglionic margin (normalized axon lengths $\mathrm{MACl}$ and $\mathrm{MAC2}$ ) in $\mathrm{ACh}-$ treated as in untreated embryos, only in untreated embryos did almost all MAC axons eventually reach the next anterior ganglion and fasciculate with the PC axon (normalized axon length
MAC4), whereas in ACh-treated embryos most MAC axons only remained as short stumps (MAC1, Fig. $8 C$ ). The complementary situation obtained for the LAC axon, for which there was no early outgrowth in either ACh-treated or untreated embryos (corresponding to the normalized axon length LAC0). But almost all Retzius neurons in ACh-treated embryos had acquired $\mathrm{LAC}$ axons that reached the next anterior ganglion and fasciculated with the PC axons (normalized axon length I.AC3, Fig. $10 C$ ), whereas very few did so in untreated embryos.

Sensitive period. To ascertain whether there is a sensitive period to which the induction of MAC axon retraction by $\mathrm{ACh}$ treatment is limited, embryos were subjected to a $24 \mathrm{hr}$ pulse of ACh treatment at various embryonic stages, ranging from stage $9(0 / 4)$ to stage $10(0 / 5)$. After the ACh pulse, the embryos were rinsed and transferred to normal medium and cultured for 1 to 4 more $\mathrm{d}$ before being fixed and stained immunohistochemically. The $24 \mathrm{hr}$ ACh pulse induced the disappearance of MAC axons as early as $t_{\mathrm{R}}=-0.5 \mathrm{~d}$ and as late as $t_{\mathrm{R}}=2 \mathrm{~d}$. However, the degree of sensitivity was not uniformly distributed over this period; the peak induction sensitivity occurred at $t_{\mathrm{R}}=1.5 \mathrm{~d}$ (Fig. $11 A$ ). This time of peak sensitivity just preceded the first ACh treatment-induced morphological changes of the Retzius neuron (Fig. 6C).

Kinetics of induction. To determine the kinetics of the induc-

Figure 6. Average axon lengths in Retzius neurons at the normalized Retzius neuron ages $t_{\mathrm{R}}$. Only the right-hand member of the Retzius neuron pair in each of the two adjacent ganglia is shown. The outline of the third ganglion, whose Retzius neuron has been omitted, is only partially drawn as a reference for the PC axon and its branches. Developmental changes in the relative distance between ganglia have been omitted. This drawing is based on the series of immunohistochemically stained embryos, except for the projections into adjacent ganglia, which are based on one Lucifer yellow-injected embryo. $D P$, axon in DP nerve; $M A$, axon in MA nerve; $P C$, axon in posterior connective nerve; cn, connective nerve; gm, ganglionic margin. $A$, Retzius neurons with a persistent MAC axon. $B$, Retzius neurons in which the MAC axon is replaced by an LAC axon. $C$, Retzius neurons in ACh-treated embryos. Note the initial growth and subsequent disappearance of the temporary MAC axon in $B$ and $C$. In untreated embryos $(B)$, the MAC axon disappears at a normalized age of more than $4 \mathrm{~d}$, while in ACh-treated embryos $(C)$ the MAC axon disappears at a normalized age of about $1.5 \mathrm{~d}$ (unlabeled open arrow). 


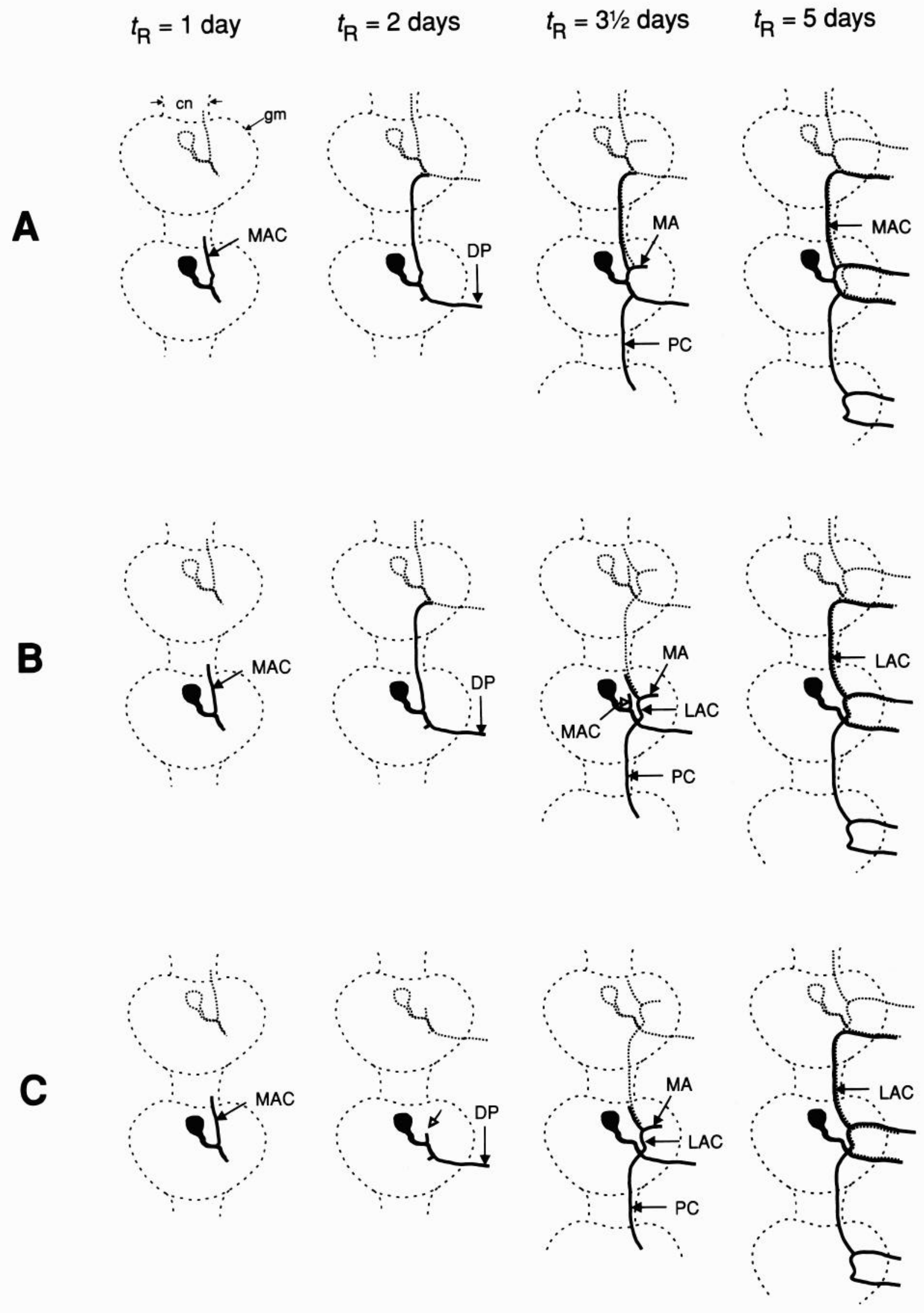



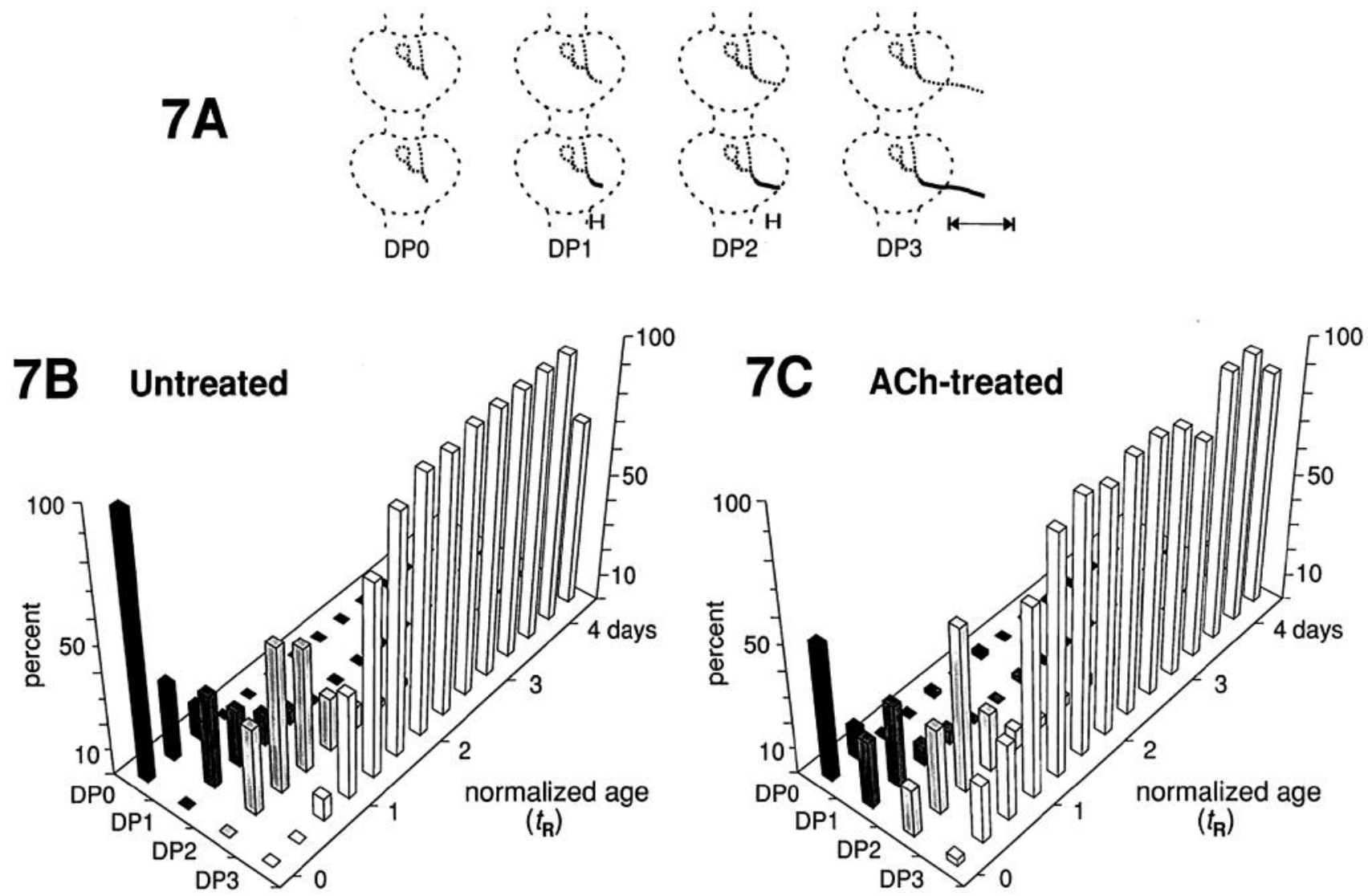

8A
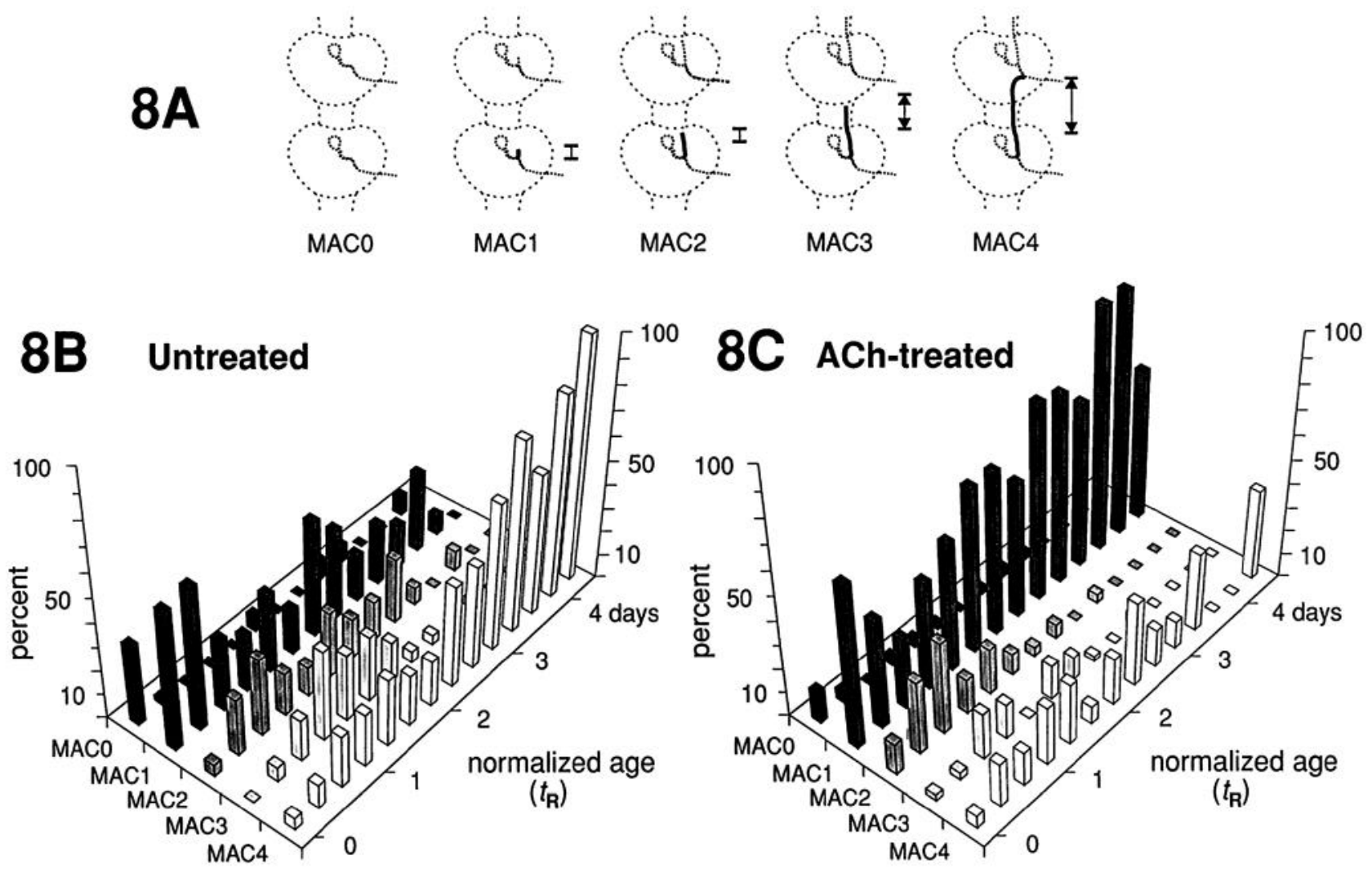
tion process during the period of peak sensitivity, stage $9(3 / 4)$ embryos were exposed to $\mathrm{ACh}$ treatment for various numbers of hours, then rinsed and transferred to normal medium and fixed $24 \mathrm{hr}$ after the beginning of the treatment. This experiment showed that a minimum of $8 \mathrm{hr}$ exposure to ACh treatment was needed for inducing the disappearance of a significant fraction of MAC axons. This effect increased until a $100 \%$ response was reached with $96 \mathrm{hr}$ of exposure (Fig. $11 B$ ).

Dose-response. The dose-response characteristics of the induction of MAC axon disappearance by $\mathrm{ACh}$ treatment were determined by exposing stage $9(1 / 4)$ embryos to varying concentrations of acetylcholine and physostigmine for 4 or $5 \mathrm{~d}$. In addition to blocking cholinesterases and thereby enhancing the action of endogenous acetylcholine, physostigmine also has intrinsic activity at nicotinic (Somani and Dube, 1989; Albuquerque et al., 1988) as well as muscarinic (Misgeld et al., 1989) acetylcholine receptors.

In one experiment, the concentration of physostigmine was held constant at $10 \mu \mathrm{M}$, and the concentration of $\mathrm{ACh}$ was varied from 0 to $10 \mathrm{mM}$ (Fig. $11 \mathrm{C}$ ). Physostigmine at $10 \mu \mathrm{M}$ induced the disappearance of MAC axons even in the absence of added $\mathrm{ACh}$. The inductive effect increased with increasing concentrations of $\mathrm{ACh}$, until a maximum induction was reached at about $1 \mathrm{mM}$ ACh.

In another experiment, the concentration of $\mathrm{ACh}$ was held constant at $1 \mathrm{mM}$, and the concentration of physostigmine was varied from 0 to $100 \mu \mathrm{M}$ (Fig. 11D). No significant MAC axon disappearance was induced by physostigmine concentrations below $1 \mu \mathrm{M}$, but above that level increasing levels of physostigmine increased the inductive effect, until a maximum induction was reached at $100 \mu \mathrm{M}$.

In addition to the induction of MAC axon disappearance, a progressive increase in 5-HT immunoreactivity of Retzius neurons was observed upon exposure of the embryos to increasing doses of both acetylcholine and physostigmine.

Induction of MAC axon retraction by ACh treatment. At least three hypotheses can account for the early disappearance of the MAC axon induced by ACh treatment: (1) Its loss of capacity to react with anti-5-HT antibody and, thus, failure to stain in the immunohistochemical procedure. (2) Its physical disintegration. (3) Its retraction to the branch point at which it originated. Of these three alternatives, only the retraction hypothesis predicts the collapse of growth cones (Bandtlow et al., 1993; Igarashi et al., 1993) at the MAC axon tip, and that MAC axons that were present prior to the onset of $\mathrm{ACh}$ treatment become shorter during the treatment, before they eventually disappear altogether.

This prediction was tested by the following experiment. Stage 9(2/4) embryos were exposed to ACh treatment, and, $24 \mathrm{hr}$ later, were fixed at stage $9(3 / 4)$. Other, untreated control specimens were fixed either at stage $9(2 / 4)$ or at stage $9(3 / 4)$. All specimens were stained immunohistochemically and scored for MAC growth cone size and normalized MAC axon lengths (Table 2).

At stage $9(2 / 4)$, prior to the onset of ACh treatment, $59 \%$ had MAC axons with large or intermediate size growth cones with visible filopodia, such as one of the two neurons shown in Figure $3 A$, and $85 \%$ of the Retzius neurons had MAC axons which had already reached or transcended the ganglionic margin. By stage $9(3 / 4)$, there was a slight decrease in the size of growth cones (compare Fig. 3B), while MAC axon length had increased in the untreated embryos. In the ACh-treated stage 9(3/4) embryos, however, the MAC growth cones had largely disappeared (Fig. $3 C$ ), while the MAC axons were very much shorter than in the stage $9(2 / 4)$ embryos that had been fixed prior to the onset of ACh treatment. The differences between untreated stage 9(3/4) embryos and ACh-treated stage 9(3/4) embryos were statistically significant for both Growth cone size and MAC Length $(p<$ $0.001)$.

Hence, we conclude that ACh treatment induces the retraction of MAC axons rather than their loss of 5-HT immunoreactivity or physical disintegration.

\section{Discussion}

In support of one hypothesis that we set out to test, namely, that the retraction of the MAC axon branch of the Retzius neuron is due to its depolarization by $\mathrm{ACh}$, we found that exposure of $T$. rude embryos to ACh during a sensitive period of axon development increases the frequency of MAC axon retraction. However, at least two alternative explanations should be considered for the ACh-induced retraction of the MAC axon branch that would not attribute the observed effects to depolarization of the Retzius neuron. One of these is that ACh does not act on the Retzius neuron directly to produce the effect and that, instead, the ACh target is actually another cell, which, via some transcellular route, induces MAC axon retraction. This explanation seems unlikely, because the only cells in the leech embryo known to be ACh sensitive other than the Relzius neuruns are the body wall muscle fibers and a few central neurons (Sargent et al., 1977; Pellegrino and Simonneau, 1984). None of these

$\leftarrow$

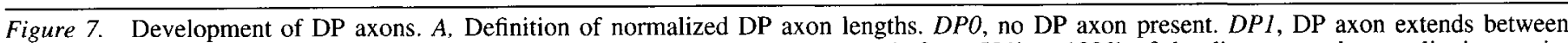

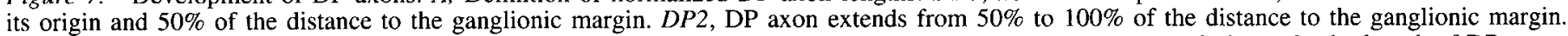

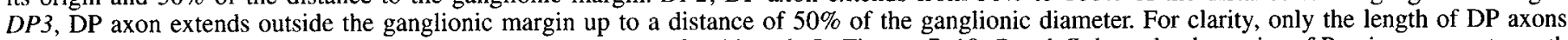

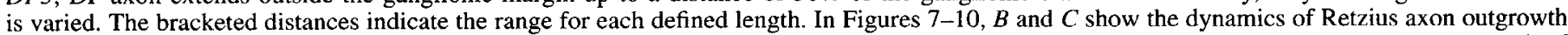

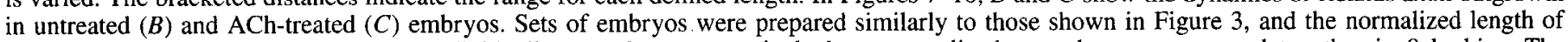

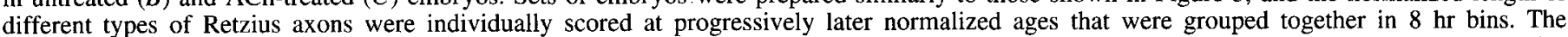

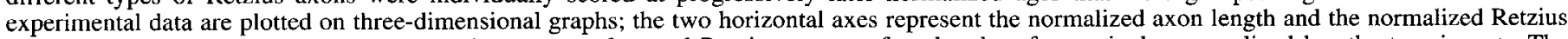

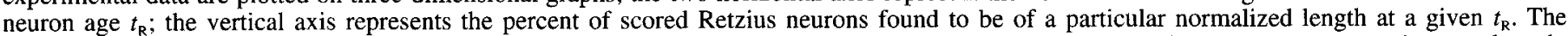

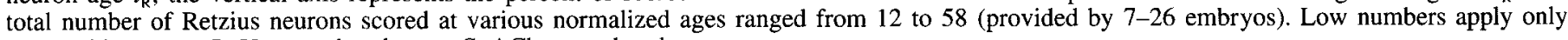
to the oldest ages. $B$, Untreated embryos. $C$, ACh-treated embryos.

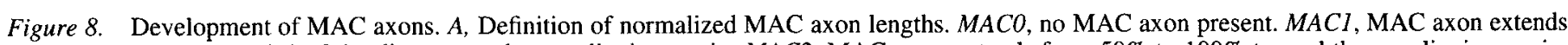

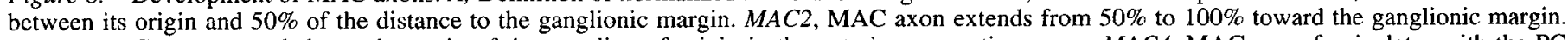

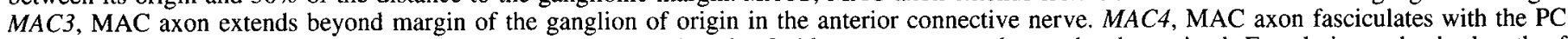

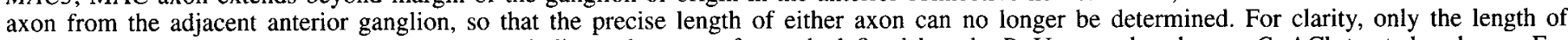

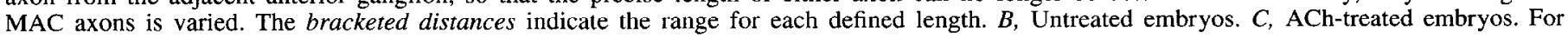
more details, see Figure 7 legend. 

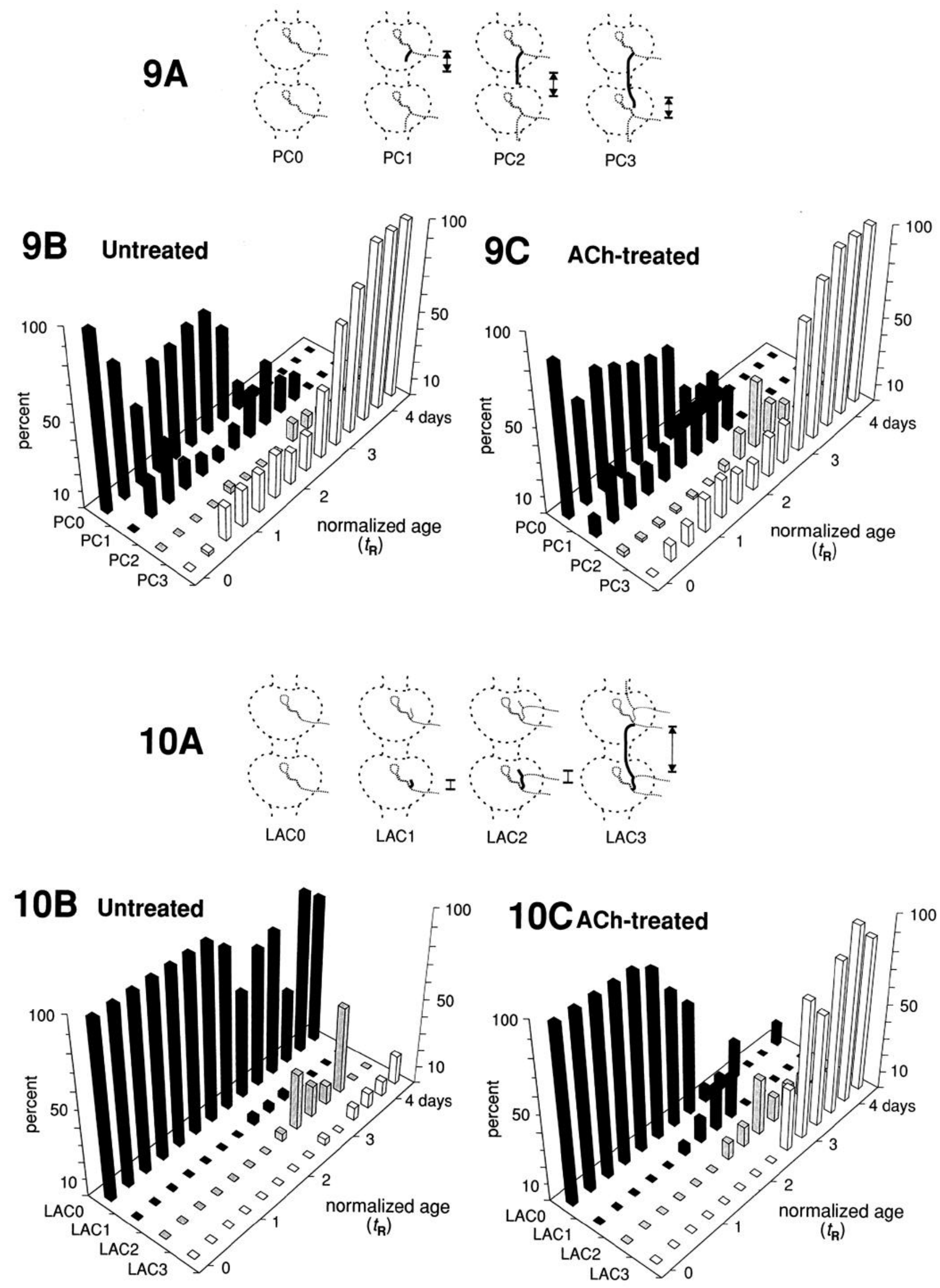
Table 2. Effects of ACh treatment on growth cone morphology and axon length of MAC axons

\begin{tabular}{llllr} 
& \multicolumn{2}{c}{ Untreated } & ACh-treated \\
\cline { 3 - 5 } & & stage $9(2 / 4)$ & stage 9 (3/4) & stage $(3 / 4)$ \\
\hline Growth cone size & GS0 & $12 \% \pm 2.7(11)$ & $11 \% \pm 1.9(7)$ & $59 \% \pm 7.7(63)$ \\
& GS1 & $11 \% \pm 3.8(11)$ & $17 \% \pm 6.1(11)$ & $19 \% \pm 4.5(20)$ \\
& GS2 & $28 \% \pm 5.5(27)$ & $27 \% \pm 5.1(17)$ & $9 \% \pm 3.8(10)$ \\
& GS3 & $31 \% \pm 6.3(28)$ & $17 \% \pm 1.2(11)$ & $3 \% \pm 1.8(3)$ \\
MAC length & Fasciculated & $18 \% \pm 5.6(16)$ & $27 \% \pm 7.6(17)$ & $11 \% \pm 3.4(11)$ \\
& MAC0 & $3 \% \pm 1.4(3)$ & $2 \% \pm 1.7(1)$ & $13 \% \pm 6.1(14)$ \\
& MAC1 & $12 \% \pm 6.5(12)$ & $11 \% \pm 6.6(7)$ & $43 \% \pm 7.5(47)$ \\
& MAC2 & $52 \% \pm 3.5(50)$ & $38 \% \pm 8.9(24)$ & $28 \% \pm 4.6(30)$ \\
& MAC3 & $15 \% \pm 6.0(15)$ & $22 \% \pm 6.4(14)$ & $5 \% \pm 2.2(5)$ \\
& MAC4 & $18 \% \pm 5.6(16)$ & $27 \% \pm 7.6(17)$ & $11 \% \pm 3.4(11)$
\end{tabular}

Five stage 9(2/4) specimens of one batch of embryos were exposed to $1 \mathrm{mM} \mathrm{ACh}$ and $10 \mu \mathrm{M}$ physostigmine for 24 $\mathrm{hr}$ and fixed at stage $9(3 / 4)$. Of eight other control specimens from the same batch that were not exposed to ACh treatment, five were fixed at stage $9(2 / 4)$, and three at stage $9(3 / 4)$. Data are expressed as percentage of scored Retzius neurons, mean \pm SEM, from segments M1-M11. The absolute number of scored Retzius neurons is given in parentheses; the total number of neurons present was 110 in untreated stage $9(2 / 4)$ and in ACh-treated embryos, and 66 in untreated stage $9(3 / 4)$. FAt stage $9(2 / 4)$, prior to ACh treatment, neurons in these segments had normalized ages $t_{\mathrm{R}}$ from 0 to $1.5 \mathrm{~d} ; 24 \mathrm{hr}$ later, at stage $9(3 / 4)$, ages ranged from 1 to $2.5 \mathrm{~d}$. Over this range of ages, the reproductive Retzius neurons do not differ from the standard Retzius neurons.] All specimens were stained immunohistochemically and scored for MAC axon lengths as defined in Figure 8A. Growth cone size was scored by visual estimate: GS0, thin, pointed axon tip with no growth cone visible (unlabeled filled arrow, Fig. $3 \mathrm{C}$ ); GS1, blunt axon ending with minimal growth cone and no filopodia visible; GS2, growth cone of intermediate surface area and/or visible filopodia (unlabeled open arrows, Fig. 3B); GS3, growth cone with a large surface area and numerous filopodia (unlabeled open arrow, Fig. 3A). In MAC axons that had fasciculated with the adjacent anterior PC axon, no growth cone was visible. $\chi^{2}$ tests were performed according to Hassard (1991). The differences between untreated stage 9(3/4) embryos and ACh-treated embryos were statistically significant for both growth cone size $(p<0.001$, $\left.\chi^{2}=46.83\right)$ and MAC length $\left(p<0.001, \chi^{2}=38.29\right)$.

cells have been described to make direct contact with Retzius neurons.

Another alternative explanation is that $\mathrm{ACh}$ does act directly on Retzius neurons but it induces MAC axon retraction via a route that does not involve depolarization. This alternative is especially cogent because we were unable to show that ACh caused depolarization at the developmental stage studied, since taking electrical recordings from developing Retzius neurons in T. rude embryos proved to be impossible, due to their small size.

The prolonged exposure to $\mathrm{ACh}$ that was necessary to induce MAC axon retraction suggests that the response was most likely mediated by a nondesensitizing non-nicotinic $\mathrm{ACh}$ receptor. Non-nicotinic receptors in the leech have not yet been fully characterized (Kristan et al., 1993; Szczupak et al., 1993). It is possible that, like vertebrate muscarinic receptors, they mediate the inhibition of adenylyl cyclase and the stimulation of phospholipase $\mathrm{C}$, in addition to changes in membrane polarization. Thus, the effect of ACh on axon retraction may, in fact, be caused independent of a change in membrane polarization.

It is also plausible that the Retzius neurons were actually depolarized by $\mathrm{ACh}$ in this study. In embryonic Retzius neurons, the beginning of axon outgrowth coincides with the onset of electrical excitability (Gottmann et al., 1989; Schirrmacher et al., 1991). About 2 d later, and about $3 \mathrm{~d}$ before the Retzius axons contact the gonadal tissues, the first electrical responses to 5-HT can be recorded from embryonic Retzius neurons (Lessmann and Dietzel, 1991). Thus, it seems likely that their electrical responses to $\mathrm{ACh}$ begin at about the same time, in agreement with our finding that exposure to $\mathrm{ACh}$ begins to have an effect on axon retraction at about $2 \mathrm{~d}$ after the onset of axon outgrowth. The earliest recordings of electrical responses to ACh in embryonic Retzius neurons, made about a week later in devclopment, showed them to be virtually identical to the adult responses, including a slow, nondesensitizing depolarization of a non-nicotinic nature (Kristan et al., 1993).

\section{Neurotransmitters and specificity in axonal pruning}

In the development of both vertebrate and invertebrate nervous systems, many axons and axon collaterals are pruned to allow the formation of function-specific (Simon and O'Leary, 1992) and species-specific (Stanfield et al., 1987) neuronal connections. For example, neurons of layer 5 in all areas of the developing rat neocortex initially project axon collaterals to a set of

$$
\leftarrow
$$

Figure 9. Development of PC axons. A, Definition of PC axon lengths. PCO, no PC axon present. PC1, PC axon extends between its origin and the ganglionic margin. $P C 2, \mathrm{PC}$ axon extends outside ganglion of origin into the posterior connective nerve. $P C 3, \mathrm{PC}$ axon fasciculates with the MAC axon from the adjacent posterior ganglion, so that the precise length of either axon can no longer be determined. For clarity, only the length of PC axons is varied. The bracketed distances indicate the range for each defined length. B, Untreated embryos. C, ACh-treated embryos. For more details, see Figure 7 legend.

Figure 10. Development of LAC axons. A, Definition of LAC axon lengths. $L A C O$, no LAC axon present. $L A C I$, LAC. axon extends between its origin and 50\% toward the future branch point of the MA axon. $L A C 2$, LAC axon extends between LAC1 and $50 \%$ from MA branch point toward the ganglionic margin. $L A C 3$, LAC axon fasciculates with the PC axon from the adjacent anterior ganglion, so that the precise length of either axon can no longer be determined. For clarity, PC axons are not shown in this drawing. The bracketed distances indicate the range for each defined length of the LAC axon. $B$, Untreated embryos. $C$, ACh-treated embryos. For more details, see Figure 7 legend. 

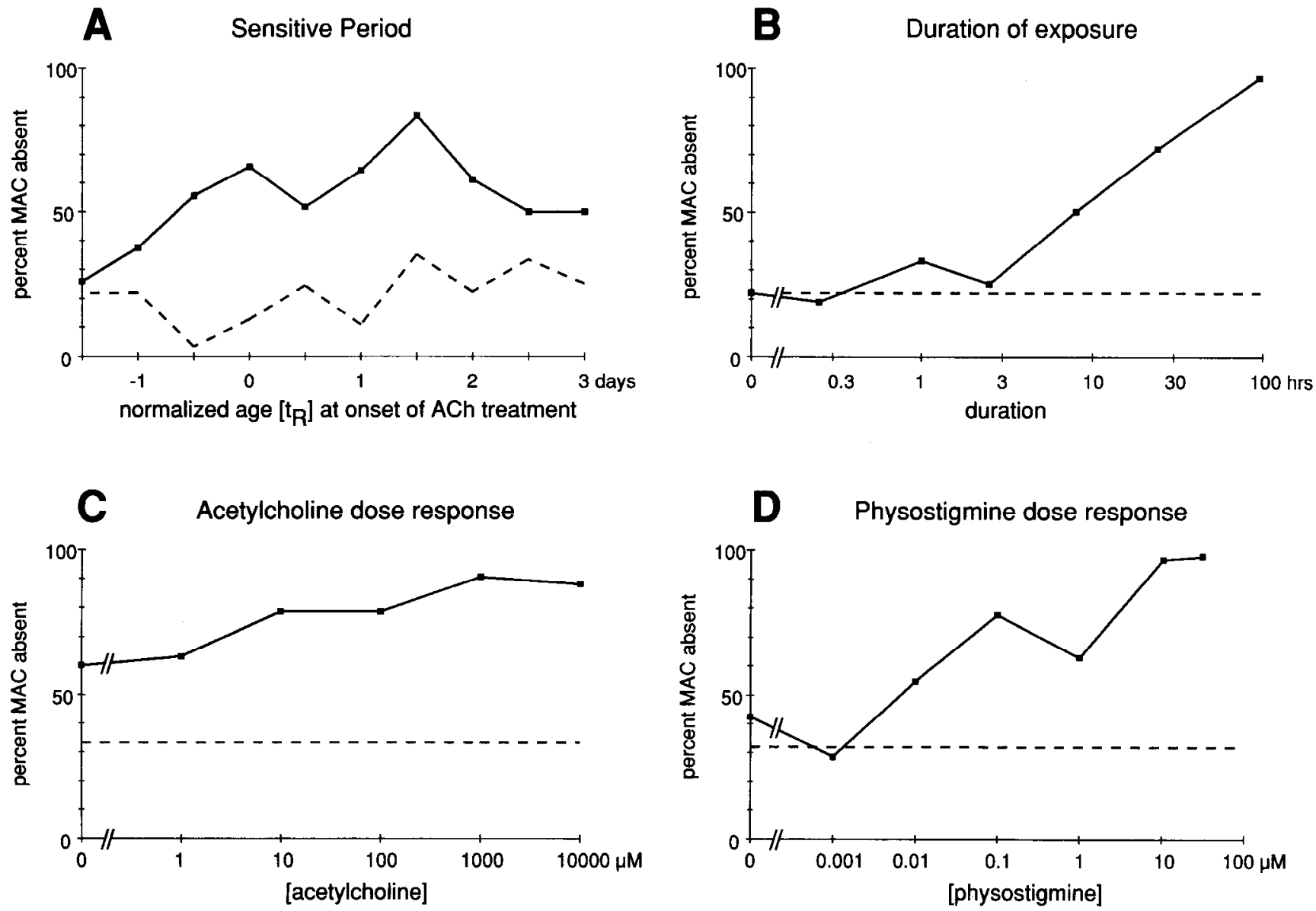

Figure 11. Sensitive period, duration of exposure, and dose responses for acetylcholine and physostigmine. Data are expressed as the percentage of scored Retzius neurons with an MAC axon length of MACO or MAC1 (as defined in Fig. 8A). The dotted line indicates the baseline in untreated embryos; in $B, C$, and $D$ it is equivalent to one data point that does not correspond to a value on the $\mathrm{X}$-axis. $A$, Sensitive period of response to acetylcholine treatment. Nine embryos were exposed to $1 \mathrm{~mm}$ ACh and $10 \mu \mathrm{M}$ physostigmine for $24 \mathrm{hr}$, beginning at stages ranging from $9(0 / 4)$ to $10(0 / 5)$, rinsed at the end of ACh treatment, and fixed at stages ranging from $9(3 / 4)$ to $10(3 / 5)$, according to the following (fixation stage: stage at treatment onset, number of embryos examined). $9(3 / 4): 9(0 / 4), 1 ; 9(1 / 4), 1 ; 9(2 / 4), 1.10(0 / 5): 9(0 / 4), 1 ; 9(1 / 4), 1 ; 9(2 / 4), 1 ; 9(3 / 4), 1.10(3 / 5)$ : $9(3 / 4), 1 ; 10(0 / 5), 1$. Each data point represents 9-36 Retzius neurons from one to six immunohistochemically stained embryos; the normalized ages $t_{\mathrm{R}}$ at the time of fixation ranged from 1.5 to $6.5 \mathrm{~d}$. Low numbers apply only to the oldest ages. (ACh treatment does not affect the normalized Retzius age definition; see Fig. 5). Data are plotted against normalized age $t_{\mathrm{R}}$ at the beginning of treatment. The dotted control baseline was obtained by subjecting three untreated embryos, one each fixed at stages $9(3 / 4), 10(0 / 5)$, and $10(3 / 5)$, to the same calculation procedure. $B$, Dependence of the acetylcholine response on duration of exposure. Stage $9(3 / 4)$ embryos were exposed to $1 \mathrm{mM}$ ACh and $10 \mu \mathrm{M}$ physostigmine for a varying number of hours and then immediately rinsed. All specimens were fixed $24 \mathrm{hr}$ after the beginning of the treatment. Embryos treated for $96 \mathrm{hr}$ were fixed at the same time to allow the comparison of neurons of a similar age, but ACh treatment was begun earlier at stage $9(1 / 4)$. Each data point represents 5156 Retzius neurons from two embryos, with normalized ages $t_{\mathrm{R}}$ ranging from 2.5 to $4.5 \mathrm{~d}$. $C$, Dose response to acetylcholine in the presence of a constant physostigmine concentration. Stage $9(1 / 4)$ embryos were exposed to varying concentrations of acetylcholine at a constant concentration of $10 \mu \mathrm{M}$ physostigmine for $5 \mathrm{~d}$. Embryos were rinsed and fixed at stage $10(2 / 5)$. For each data point, $28-42$ Retzius neurons with nornulized ages $t_{\mathrm{R}}$ ranging from 2.5 to $5.5 \mathrm{~d}$ from one stained embryo were scored. Physostigmine elicits a response even in the absence of acetylcholine. D, Dose response to physostigmine in the presence of a constant acetylcholine concentration. Stage $9(1 / 4)$ embryos were exposed to varying concentrations of physostigmine at a constant concentration of $1 \mathrm{~mm}$ acetylcholine for $4 \mathrm{~d}$. Embryos were rinsed and fixed at stage $10(1 /$ 5). For each data point, 27-33 Retzius neurons with normalized ages $t_{\mathrm{R}}$ ranging from 1.5 to $4.5 \mathrm{~d}$ from 1 embryo were scored.

targets including the superior colliculus, the inferior olive, and the dorsal column nuclei of the spinal cord. In later development, layer 5 neurons in the motor cortex lose their collateral projections to the superior colliculus, while those in the visual cortex lose their collateral projections to the inferior olive and the dorsal column nuclei (O'Leary et al., 1990; O’Leary, 1992).

The mechanisms for this kind of selective axon pruning have not yet been elucidated. However, since neuronal activity is important in the sculpting of neuronal connections (ConstantinePaton et al., 1990; Shatz, 1990), neurotransmitters are primary candidates for regulatory agents in this process. Studies of neurons in cell culture have indicated that neurotransmitters can inhibit axon outgrowth (Cohan and Kater, 1986; McCobb and Kater, 1988; Mattson, 1988; Lipton and Kater, 1989; Schmidt and Kater, 1993). More specifically, acetylcholine has been shown to induce neurite retraction in cultured embryonic chick ciliary ganglion cells (Pugh and Berg, 1994). The present study provides an example of specificity in axonal pruning in the intact developing embryo mediated by acetylcholine.

\section{Axon branch specificity}

How is it possible that, as found in the present study, exposure of leech embryos to ACh causes retraction of only the MAC. axon branch without affecting other Retzius axon branches? 
The most likely explanation of such a specific, localized effect is that responses of growing axon branches to particular stimuli depend on their microenvironment (Spira et al., 1987). A comparison of the growth behavior of the MAC, LAC, and PC axon branches in the present study suggests that, among them, the MAC branch appears to be exposed to a microenvironment that promotes an early and rapid outgrowth, which is, however, susceptible to perturbations, whereas PC and LAC branches appear to be exposed to a microenvironment that promotes a delayed but stable outgrowth pattern.

Microenvironmental factors influencing the outgrowth of Retzius axons have been identified in cell culture experiments. In cell culture, Retzius neurons extend axonal branches much faster and more elaborately on a laminin-rich leech extracellular matrix than on a Concanavalin A substrate (Masuda-Nakagawa and Nicholls, 1991). Moreover, axonal branches of Retzius neurons maintained on a leech-laminin-rich extracellular matrix are particularly susceptible to perturbations of their growth, since depolarization causes their retraction only on leech extracellular matrix, but not on a Concanavalin A substrate (GrumbacherReinert and Nicholls, 1992; Neely, 1993). This difference is probably duc to the greater number of functional $\mathrm{Ca}^{2+}$ channels in Retzius neuron processes growing on leech laminin than on Concanavalin A (Ross et al., 1988; Masuda-Nakagawa and Nicholls, 1991). Laminin is expressed in the connective nerves and ganglionic neuropil of leeches only during embryonic development (Masuda-Nakagawa and Nicholls, 1991).

Hence, the axon branch-specific effect of ACh treatment observed in the present study may be attributable to laminin or other adhesion molecules of the extracellular matrix of the medial pathway in the ganglionic neuropil, which only the MAC branch among the Retzius axon branches is following, and which could render the MAC branch uniquely susceptible to a growth-regulating, depolarizing signal.

\section{Significance for normal Retzius neuron development}

We found that in untreated embryos a spontaneous disappearance of the M $\mathrm{MC}$ axon branch occurred primarily in the reproductive segments M5 and M6. Furthermore, in Retzius neurons of the reproductive segments, a transient increase in 5-HT content was observed similar to that found in Retzius neurons of standard midbody segments upon their exposure to ACh. These findings suggest that ACh treatment may have induced a process in standard midbody segments similar to that which occurs in normal development of the reproductive segments.

Most previous studies of Retzius neuron development involved the species Hirudo medicinalis, where, in contrast to the species studied here ( $T$. rude), both anterior and posterior connective axon branches of the Retzius neuron are retracted, and no additional axon (like $\mathrm{LAC}$ ) is formed to replace them. A representative Retzius neuron of a standard midbody segment in adult $H$. medicinalis is shown in Figure $2 D$.

In these studies, the disappearance of Retzius axon branches in the connective nerves of the reproductive segments has been shown to depend on the contact of the peripheral Retzius axon branches with their target, the gonadal tissues (Jellies et al., 1987; Loer et al., 1987; Loer and Kirstan, 1989; French et al., 1992); in T. rude, these have been shown to be of mesodermal origin (Gleizer and Stent, 1993). Target contact can induce depolarization and other changes in cultured Retzius neurons (Fernandez de Miguel et al., 1992; Cooper et al., 1992). In summary, it appears possible that ACh treatment may have simulated a process that normally contributes to the segmental differentiation of the Retzius neuron.

\section{References}

Albuquerque EX, Aracava Y, Cintra WM, Brossi A, Schönenberger B, Deshpande SS (1988) Structure-activity relationship of reversible cholinesterase inhibitors: activation, channel blockade and stereospecificity of the nicotinic acetylcholine receptor-ion channel complex. Braz J Med Biol Res 21:1173-1196.

Bandtlow CE, Schmidt MF, Hassinger TD, Schwab ME, Kater SB (1993) Role of intracellular calcium in NI-35-evoked collapse of neuronal growth cones. Science 259:80-83.

Baptista CA, Macagno ER (1988) Modulation of the pattern of axonal projections of a leech motor neuron by ablation or transplantation of its target. Neuron 1:949-962.

Baptista CA, Gershon TR, Macagno ER (1990) Peripheral organs control central neurogenesis in the leech. Nature 346:855-858.

Braun J, Stent GS (1989) Axon outgrowth along segmental nerves in the leech. I. Identification of candidate guidance cells. Dev Biol 132: $471-485$.

Cohan CS, Kater SB (1986) Suppression of neurite elongation and growth cone motility by electrical activity. Science $232: 1638-1640$.

Cohan CS, Connor JA, Kater SB (1987) Electrically and chemically mediated increases in intracellular calcium in neuronal growth cones. J Neurosci 7:3588-3599.

Constantine-Paton M, Cline HT, Debski E (1990) Patterned activity, synaptic convergence, and the NMDA receptor in developing visual pathways. Annu Rev Neurosci 131:129-154.

Cooper RL, Fernandez de Miguel F, Adams WB, Nicholls JG (1992) Anterograde and retrograde effects of synapse formation on calcium currents and neurite outgrowth in cultured leech neurons. Proc R Soc Lond [Biol] 249:217-222.

Fernandez J (1980) Embryonic development of the glossophoniid leech Theromyzon rude: characterization of developmental stages. Dev Biol 76:245-262.

Fernandez de Miguel F, Cooper RL, Adams WB (1992) Synaptogenesis and calcium current distribution in cultured leech neurons. Proc R Soc Lond [Biol] 247:215-221.

French KA, Jordan SM, Loer CM, Kristan WB Jr (1992) Mesenchyme of embryonic reproductive ducts directs process out growth of Retzius neurons in the medicinal leech. Dev Biol 153:122-140.

Gao WQ, Macagno ER (1987a) Extension and retraction of axonal projections by some developing neurons in the leech depends upon the existence of neighboring homologues. I. The HA cells. J Neurobiol 18:43-59

Gao WQ, Macagno ER (1987b) Extension and retraction of axonal projections by some developing neurons in the leech depends upon the existence of neighboring homologues. II. The AP and AE neurons. J Neurobiol 18:295-313.

Gleizer L, Stent GS (1993) Developmental origin of segmental identity in the leech mesoderm. Development 117:177-189.

Glover JC (1987) Serotonin storage and uptake by identified neurons in the leech Haementeria ghilianii. J Comp Neurol 256:117-127.

Glover JC, Mason A (1986) Morphogenesis of an identified leech neuron: segmental specification of axonal outgrowth. Dev Biol 115:256260.

Gottmann K, Dietzel ID, Lux HD (1989) Proton-induced chloride current and voltage-activated $\mathrm{Na}^{+}$and $\mathrm{Ca}^{2+}$ currents in embryonic neurons from the medicinal leech (Hirudo medicinalis). Neurosci Lett 96:173-178.

Grumbacher-Reinert S, Nicholls J (1992) Influence of substrate on retraction of neurites following electrical activity of leech Retzius cells in culture. J Exp Biol 167:1-14.

Hassard TH (1991) Understanding biostatistics, pp 100-114; 233-237. St. Louis: Mosby-Year Book.

Haydon PG, McCobb DP, Kater SB (1984) Serotonin selectively inhibits growth cone motility and the connection of identified neurons. Science 226:561-564.

Igarashi M, Strittmatter SM, Vartanian T, Fishman MC (1993) Mediation by G-proteins of signals that cause collapse of growth cones. Science 259:77-79.

Ip NY, Zigmond RE (1984) Pattern of presynaptic nerve activity can determine the type of neurotransmitter regulating a postsynaptic event. Nature 311:472-474.

Jellies J, Loer CM, Kristan WB (1987) Morphological changes in leech 
Retzius neurons after target contact during embryogenesis. J Neurosci 7:2618-2629.

Kater SB, Mills LR (1991) Regulation of growth cone behavior by calcium. J Neurosci 11:891-899.

Kater SB, Mattson M, Cohan CS, Connor J (1988) Calcium regulation of the neuronal growth cone. Trends Neurosci 11:315-321.

Kristan WB, French KA, Szczupak L (1993) Developmental regulation of segment-specific cholinergic receptors on Retzius neurons in the medicinal leech. J Neurosci 13:1577-1587.

Kuwada JY, Kramer AP (1983) Embryonic development of the leech nervous system: primary axon outgrowth of identified neurons. J Neurosci 3:2098-2111.

Lauder JM (1987) Neurotransmitters as morphogenetic signals and trophic factors. In: Model systems of development and aging of the nervous system (Vernadakis A, ed), pp 219-237. Boston: Nijhoff.

Leslie FM (1992) Neurotransmitters as neurotrophic factors. In: Neurotrophic factors (Loughlin SE, Fallon JH, eds), pp 565-598. San Diego: Academic.

Lessmann V, Dietzel ID (1991) Development of serotonin induced ion currents in identified embryonic Retzius cells from the medicinal leech (Hirudo medicinalis). J Neurosci 11:800-809.

Levi-Montalcini $R$ (1987) The nerve growth factor: thirty-five years later. EMBO J 6:1145-1154.

Lipton SA, Kater, SB (1989) Neurotransmitter regulation of ncuronal outgrowth, plasticity and survival. Trends Neurosci 12:265-270.

Loer CM, Kristan WB Jr (1989) Central synaptic inputs to identified leech neurons determined by peripheral targets. Science 244:64-66.

Loer CM, Jellies J, Kristan WB (1987) Segment-specific morphogenesis of leech Retzius neurons requires particular peripheral targets. J Neurosci 7:2630-2638.

Longo FM, Holtzman DM, Grimes M, Mobley WC (1993) Nerve growth factor: actions in the peripheral and central nervous systems. In: Neurotrophic factors (Loughlin SE, Fallon JH, eds), pp 209-256. San Diego: Academic.

Masuda-Nakagawa LM, Nicholls JG (1991) Extracellular matrix molecules in development and regeneration of the leech CNS. Philos Trans R Soc Lond [Biol] 331:323-335.

Mattson MP (1988) Neurotransinilters in the regulation of neuronal cytoarchitecture. Brain Res Rev 13:179-212.

McCobb DP, Kater SB (1988) Membrane voltage and neurotransmitter regulation of neuronal growth cone motility. Dev Biol 130:599-609.

Misgeld U, Muller W, Polder HR (1989) Potentiation and suppression by eserine of muscarinic synaptic transmission in the guinea-pig hippocampal slice. J Physiol (Lond) 409:191-206.

Muller KJ, Nicholls JG, Stent GS, eds (1981) Neurobiology of the leech. Cold Spring Harbor, NY: Cold Spring Harbor Laboratory.

Neely MD (1993) Role of substrate and calcium in neurite retraction of leech neurons following depolarization. J Neurosci 13:1292-1301.

O'Leary DDM (1989) Do cortical areas emerge from a protocortex. Trends Neurosci 12:400-406.

O'Leary DDM (1992) Development of connectional diversity and specificity in the mammalian brain by the pruning of collateral connections. Curr Opin Neurobiol 2:70-77.

O'Leary DDM, Bicknese AR, De Carlos JA, Heffner CD, Koester SE, Kutka LJ, Terashima T (1990) Target selection by cortical axons: alternative mechanisms to establish axonal connections in the developing brain. Cold Spring Harbor Symp Quant Biol 55:453-468.

Pellegrino M, Simonneau M (1984) Distribution of receptors for acetylcholine and 5-hydroxytryptamine on identified leech neurones growing in culture. J Physiol (Lond) 352:669-684.

Pugh PC, Berg DK (1994) Neuronal acetylcholine receptors that bind $\alpha$-bungarotoxin mediate neurite retraction in a calcium-dependent manner. J Neurosci 14:889 896.
Rakic P (1988) Specification of cerebral cortical areas. Science 241: 170-176.

Ross WN, Arechiga H, Nicholls JG (1988) Influence of substrate on the distribution of calcium channels in identified leech neurons in culture. Proc Natl Acad Sci USA 85:4075-4078.

Saito S, Komiya Y, Igarashi M (1991) Muscarinic acetylcholine receptors are expressed and enriched in growth cone membranes isolated from fetal and neonatal rat forebrain: pharmacological demonstration and characterization. Neuroscience 45:735-745.

Sargent PB, Yau KW, Nicholls JG (1977) Extrasynaptic receptors on cell bodies of neurones in the central nervous system of the leech. $J$ Neurophysiol 40:446-452.

Schimmacher K, Deitmer JW (1991) Sodium- and calcium-dependent excitability of embryonic leech ganglion cells in culture. J Exp Biol $155: 435-454$.

Schmidt MF, Kater SB (1993) Fibroblast growth factors, depolarization, and substratum interact in a combinatorial way to promote neuronal survival. Dev Biol 158:228-237.

Shatz CJ (1990) Impulse activity and the patterning of connections during CNS development. Neuron 5:745-756.

Simon DK, O'Leary DDM (1992) Development of topographic order in the mammalian retinocollicular projection. J Neurosci 12:12121232.

Somani SM, Dube SN (1989) Physostigmine-an overview as pretreatment drug for organophosphate intoxication. Int J Clin Pharmacol Ther Toxicol 27:367-387.

Spira ME, Zeldes D, Hochner B, Dormann A (1987) The effects of microenvironment on the redifferentiation of regenerating neurones: neurite architecture, acetylcholine receptors and calcium channel distribution. J Exp Biol 132:111-131.

Stanfield BB, Nahin BR, O'Leary DDM (1987) A transient postmamillary component of the rat fornix during development: implications for interspecific differences in mature axonal projections. J Neurosci 7:3350-3361.

Stent GS, Weisblat DA, Blair SS, Zackson SL (1982) Cell lineage in the development of the leech nervous system. In: Neuronal development (Spitzer N, ed) pp 1-44. New York: Plenum.

Stuart DK, Blair SS, Weisblat DA (1987) Cell lineage, cell death, and the developmental origin of identified serotonin and dopamine-containing neurons in the leech. J Neurosci 7:1107-1122.

Stuart DK, Torrence SA, Law IM (1989) Leech neurogenesis. I. Po sitional commitment of neural precursor cells. Dev Biol 136:17-39.

Szczupak L, Jordan S, Kristan WB Jr (1993) Segment-specific modulation of the electrophysiological activity of leech Retzius neurons by acetylcholine. J Exp Biol 183:115-135.

Torrence SA, Law MI, Stuart DK (1989) Leech neurogenesis. II. Mesodermal control of neuronal patterns. Dev Biol 136:40-60.

Wallace BG (1984) Selective loss of neurites during differentiation of cells in the leech central nervous system. J Comp Neurol 228:149153.

Weisblat DA, Shankland M (1985) Cell lineage and segmentation in the leech. Phil Trans R Soc Lond [Biol] 312:39-56.

Weisblat DA, Harper G, Stent GS, Sawyer RT (1980) Embryonic cell lineages in the nervous system of the glossophoniid leech Helobdella triserialis. Dev Biol 76:58-78.

Weisblat DA, Kim SY, Stent GS (1984) Embryonic origins of cells in the leech Helobdella triserialis. Dev Biol 104:65-85.

Wittenberg G, Loer CM, Adamo SA, Kristan WB Jr (1990) Segmental specialization of neuronal connectivity in the leech. J Comp Physiol 167:453-459.

Wolszon LR, Macagno LR (1992) Growth cone interactions and the patterns of peripheral innervation in the leech. In: The nerve growth cone (Letourneau PC, Kater SB, Macagno ER, eds), pp 305-322. New York: Raven. 